\title{
Herziening macrofaunamaatlat R4
}

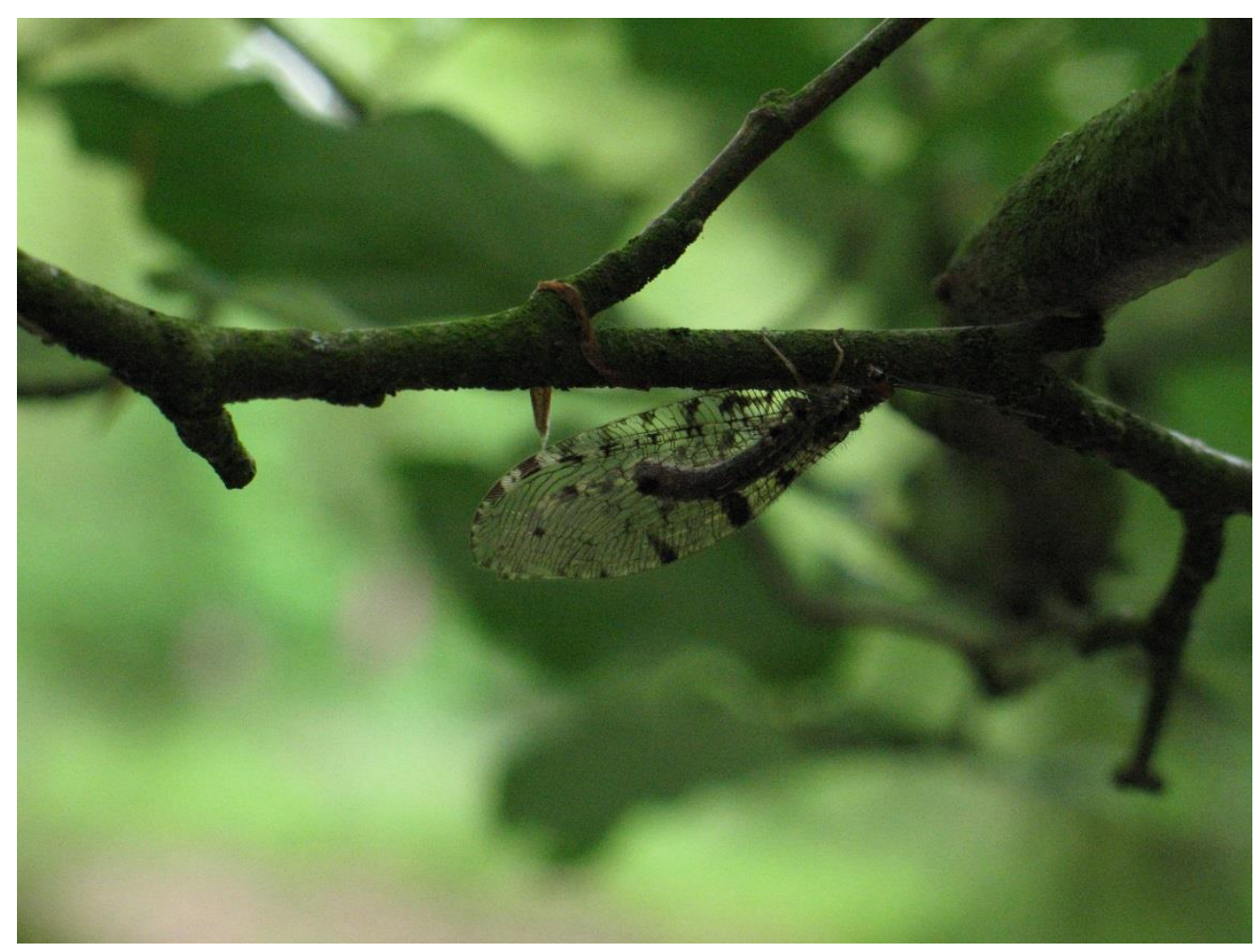

Ralf Verdonschot \& Piet Verdonschot

Notitie Zoetwatersystemen, Wageningen Environmental Research

Augustus 2018 


\author{
Auteurs \\ Ralf Verdonschot, Piet Verdonschot
}

\title{
Opdrachtgever
}

Provincie Noord Brabant, programmabureau KRW/DHZ Maasregio; contactpersoon Noud Kuijpers

\section{Projectgroep}

Marco Beers (Waterschap Brabantse Delta); Rob Fraaije (Waterschap Aa en Maas); Ron Schippers \& Mark Scheepens (Waterschap de Dommel); Barend van Maanen (Waterschap Limburg); Petra Schep (Waterschap Drents Overijsselse Delta), Bert Klutman \& Bastiaan van Zuidam (Waterschap Rijn en IJssel), Gertie Schmidt (Waterschap Vechtstromen), Harry Boonstra (Wetterskip Fryslân) m.m.v. Jeroen van Mil \& Monique Korsten (Waterschap Limburg), Hans Hop (Aqualysis waterlaboratorium).

\section{Referaat}

Verdonschot, R.C.M., Verdonschot P.F.M. (2018) Herziening macrofaunamaatlat R4. Notitie Zoetwatersystemen, Wageningen Environmental Research, Wageningen UR, Wageningen.

\section{Trefwoorden}

macrofauna, indicatoren, KRW, beoordeling, ecologische kwaliteit

\section{Beeldmateriaal}

Ralf Verdonschot

ISBN: 978-94-6343-342-6

DOI: https://doi.org/10.18174/458507

- Overname, verveelvoudiging of openbaarmaking van deze uitgave is toegestaan mits met duidelijke bronvermelding.

- Overname, verveelvoudiging of openbaarmaking is niet toegestaan voor commerciële doeleinden en/of geldelijk gewin.

- Overname, verveelvoudiging of openbaarmaking is niet toegestaan voor die gedeelten van deze uitgave waarvan duidelijk is dat de auteursrechten liggen bij derden en/of zijn voorbehouden.

Wageningen Environmental Research aanvaardt geen aansprakelijkheid voor eventuele schade voortvloeiend uit het gebruik van de resultaten van dit onderzoek of de toepassing van de adviezen 


\section{Inhoud}

1 Aanleiding en doel 3

2 Aanpak 3

2.1 Aanpassingen indicatortaxalijst 3

2.2 Vaststellen grenswaarden aangepaste maatlat 6

2. Resultaten $\quad 7$

$\begin{array}{lll}2.1 & \text { Aanpassingen indicatortaxalijst } & 7\end{array}$

2.2 Vaststellen en toetsen grenswaarden aangepaste maatlat 8

2.3 De maatlat 9

3. Discussie en aanbevelingen 11

4. Herziening tekst maatlatdocument macrofauna 12

5. Literatuur 13

Bijlage 1: Indicatorlijst 14 


\section{$1 \quad$ Aanleiding en doel}

In Noord-Brabant is geconstateerd dat de macrofaunamaatlat voor R4 beperkingen heeft voor bovenlopen met een relatief laag verhang, omdat er relatief weinig soorten voorkomen die als kenmerkend zijn aangeduid voor het KRW-type R4 (Verdonschot \& Verdonschot 2017). Dit probleem wordt door waterbeheerders ook herkend voor andere delen van Nederland.

Veel van deze in de vlakke delen ontbrekende soorten zijn in hun voorkomen beperkt tot bovenlopen of bronbeken op terras- en stuwwalranden, onder andere gekenmerkt door relatief hoge stroomsnelheid, permanente grondwatervoeding en een relatief lage en constante watertemperatuur. Vaak zijn dit beken die ontspringen als een bron, al dan niet gegraven (spreng). Deze soorten worden niet of amper aangetroffen in de bovenlopen op de hogere zandgronden in gebieden met een lager verhang. Deze beken hadden van nature gewoonlijk geen bron als oorsprong maar een veengebied waar het water diffuus (doorstroomveen of -moeras) uittrad.

De consequentie voor de beoordeling van bovenlopen met de huidige R4-maatlat is dat de 'laagland'-bovenlopen relatief slecht scoren omdat de als kenmerkend aangeduide soorten geen deel uitmaken van de regionale soortenpoule. Een bijkomend probleem is dat wanneer er maar weinig kenmerkende soorten aanwezig zijn, de maatlat weinig robuust is, wat wil zeggen dat toevalstreffers een grote rol kunnen spelen in de beoordeling.

Om deze problemen aan te pakken is de macrofaunalijst van het watertype R4 herzien, zodat deze beter bruikbaar wordt voor laaglandbeken met een relatief laag verhang en zo een beter beeld van de ecologische toestand van het waterlichaam geeft.

\section{Aanpak}

\subsection{Aanpassingen indicatortaxalijst}

Als uitgangspunt voor de herziening zijn twee databestanden gebruikt:

A. De huidige indicatorenlijst voor het watertype R4

B. Databestanden met hierin macrofaunamonsters (periode 2007 tot en met 2017) van beken aangeduid als watertype R4, aangeleverd door de verschillende waterschappen op de hogere zandgronden (in totaal 548 locaties, 1551 monsters).

De losse R4-datasets van de verschillende waterschappen zijn gecombineerd tot één bestand. Deze monsterpunten zijn vervolgens verdeeld in twee groepen aan de hand van de mate van verhang: bovenlopen in reliëfrijke gebieden en bovenlopen in relatief vlakke gebieden. In totaal gaat het om 548 R4-monsterpunten, waarvan 228 meetpunten in reliëfrijke gebieden liggen (Figuur 1).

Deze indeling is gebaseerd op de ligging van de Nederlandse hydrobiologische districten (Mol 1980) en een kaart van de fysisch-geografische regio's. Dit geeft een praktisch werkbaar beeld van de ligging van beken uit de twee groepen. Voorbeelden van reliëfrijke gebieden zijn de Pleistocene stuwwallen van de Veluwe en het pre-Pleistocene Oost Nederlands plateau en voorbeelden van relatief vlakke gebieden de Gelderse Vallei, het Kempisch Plateau en glaciale Plateau van Drenthe (Verdonschot 2000). Bijbehorend 
verhang en stroomsnelheidsgegevens van de beken worden gegeven in tabel 1 , aangevuld met twee relevante andere beektypen. Hieruit wordt direct duidelijk dat er sprake is van een gradiënt van typen van moeras naar snelstromende bovenloop.

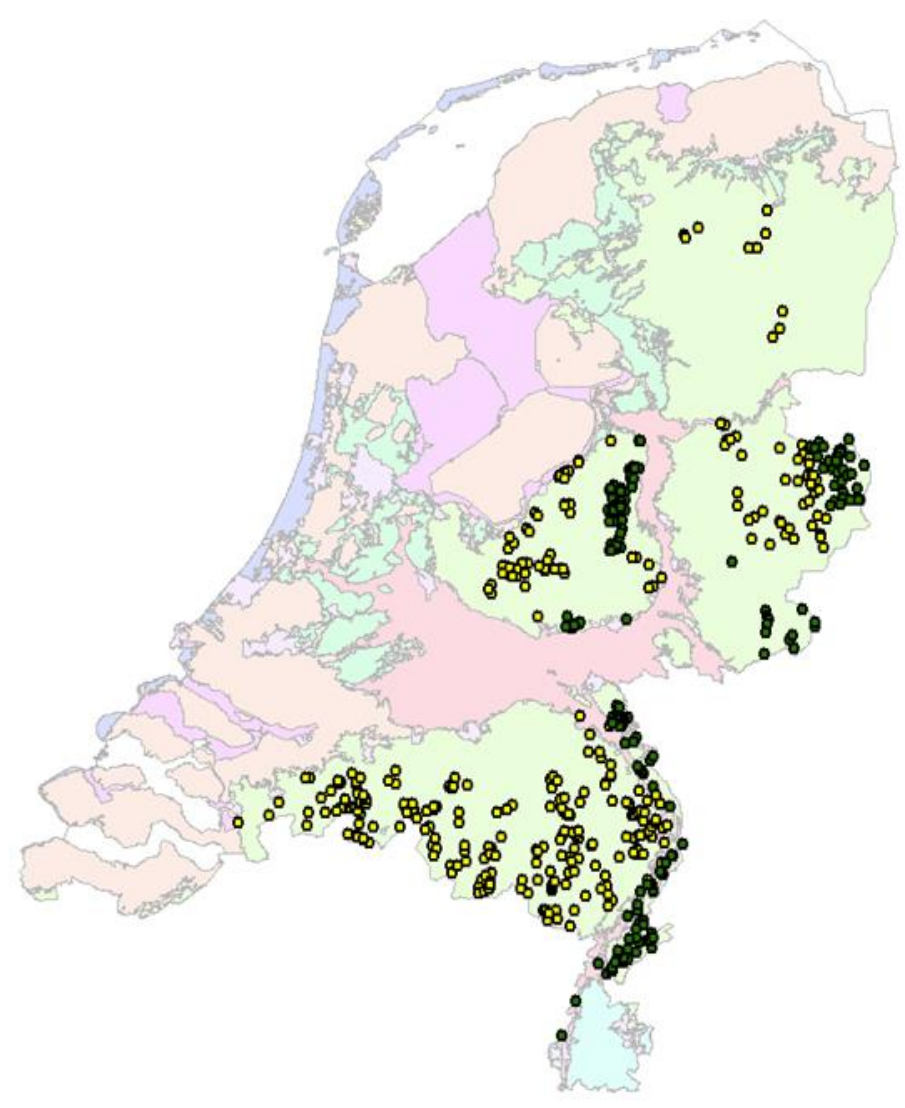

Figuur 1: Ligging R4 monsterpunten hogere zandgronden in de periode 2007-2017, geprojecteerd op de kaart met fysisch-geografische regio's in Nederland. Gele punten liggen in gebieden met een laag verhang, groene punten in reliëfrijke gebieden.

Tabel 1: Verhang en stroomsnelheden in bovenlopen op zand (Verdonschot 2000, Verdonschot et al. 2016).

\begin{tabular}{|c|c|c|}
\hline Bovenlooptype & $\begin{array}{l}\text { Verhang } \\
(\mathrm{m} / \mathbf{k m})\end{array}$ & $\begin{array}{l}\text { Stroomsnelheidsrange } \\
(\mathrm{cm} / \mathrm{s})\end{array}$ \\
\hline Doorstroommoeras & $<0.5$ & $<20$ \\
\hline $\begin{array}{l}\text { R4 Langzaam stromende bovenloop op zand, } \\
\text { subtype laag verhang }\end{array}$ & $0.5-1$ & $10-50$ \\
\hline $\begin{array}{l}\text { R4 Langzaam stromende bovenloop op zand, } \\
\text { subtype reliëfrijk }\end{array}$ & $>1$ & $30-80$ \\
\hline R13 Snelstromende bovenloop op zand & $>1$ & $>50$ \\
\hline
\end{tabular}

Vervolgens is op basis van het totale bestand een taxonlijst gegenereerd met alle taxa en de frequentie van voorkomen op de monsterpunten, verdeeld over de twee verhang-groepen. De taxonlijst is taxonomisch gecorrigeerd, bijvoorbeeld omdat oude en nieuwe namen voor dezelfde taxa in het bestand voorkwamen. De taxonlijsten zijn gehomogeniseerd op basis van de TWN-lijst. Dit leverde 1125 taxa op voor de bovenlopen in de reliëfrijke gebieden en 1337 taxa voor de bovenlopen in gebieden met een lager verhang.

De volgende stap was dat vooral alle taxa in de dataset zoveel mogelijk autoecologische informatie is bekeken. Hiervoor zijn databestanden zoals de milieu- en habitatpreferenties 
macrofauna-databestand (Verberk et al. 2012) en een breed scala aan literatuurbronnen (vaak determinatieliteratuur en verspreidingsatlassen met hierin autoecologische informatie) gebruikt. Op basis hiervan is vastgesteld of de soorten binding hebben met langzaam stromende bovenlopen, in positieve of negatieve zin om te komen tot kenmerkende, positief dominante en negatief dominante indicaties.

Voor de milieu- en habitatpreferenties kan ieder taxon in totaal 10 punten krijgen voor een parameter of sleutelfactor, welke verdeeld zijn over verschillende klassen ( bijvoorbeeld de parameter stroming heeft vijf klassen van stilstaand tot snel stromend). De verdeling is gebaseerd op de preferenties, waarbij bijvoorbeeld een taxon van snel stromende beken het grootste deel van de punten krijgt in de klasse snel stromend en geen in de klasse stilstaand. Een taxon is als indicatief (kenmerkend of positief) voor een sleutelfactor in de milieu- en habitatpreferenties beschouwd wanneer de score voor een parameter $>=5$ voor de passende klasse(n) bedraagt: ondiep (bron) (indicatief voor beken met een relatief lage watertemperatuur), matig \& snel stromend, oligotroof \& meso-oligotroof, oligosaproob en substraten hout, grind, grove detritus, zand. Idem negatief voor stilstaand, eutroof, polysaproob, niet zoet, slib.

Voor de andere literatuurbronnen werd een andere aanpak gevolgd; omdat in de verschillende bronnen nogal veel variatie in terminologie bestaat voor habitat- en milieupreferenties (er is zelden gekwantificeerde informatie voorhanden) worden in box 1 voorbeelden gegeven van indicaties die voor ons aanleiding waren een soort te selecteren. In de praktijk bleek het vaak om combinaties van de verschillende termen te gaan. Het is uitdrukkelijk de bedoeling de effecten van zo veel mogelijk stressoren mee te nemen in de indicatorlijsten voor macrofauna, zodat beter geborgd kan worden dat de beoordeling met de aangepaste soortenlijsten een relatie heeft met menselijke drukken of het wegnemen hiervan met herstelmaatregelen. De huidige soortenlijsten voor laaglandbeken zijn nu bijvoorbeeld vrij eenzijdig gericht op de factor stroming (Verdonschot \& Verdonschot 2017).

\section{Box 1: voorbeelden van inschatting indeling op basis van literatuurbronnen (inclusief vertalingen uit Engels, Duits)}

Qua habitat passend bij de typen:

bronbeken, bovenlopen, kleine beken, beekjes, sprengen, stroompjes.........

Milieuindicatie +:

Rheofiel/stroming, crenofiel/lage watertemperatuur, oligo- mesotroof, hoog

zuurstofgehalte/lage organische belasting, helder water, natuurlijke bovenlopen, specifiek habitat (mos, hout, grind), specialist, typisch voor, bijzonder

Milieuindicatie -

Verontreinigd, organisch belast, gekanaliseerd, genormaliseerd, zuurstofloos, ubiquist, weinig eisen, verstoring, gedegradeerd.

Dominant

Talrijk in, veel voorkomend, hoge aantallen........

Op basis van de gevonden informatie zijn de bestaande indicaties geëvalueerd en zijn indicatoren toegevoegd of afgevoerd. De aangepaste lijst is tenslotte beoordeeld door gebruikers en er zijn eventuele aanpassingen doorgevoerd om tot een definitieve lijst te komen. Een schematisch overzicht van de doorlopen stappen is gegeven in figuur 2. 


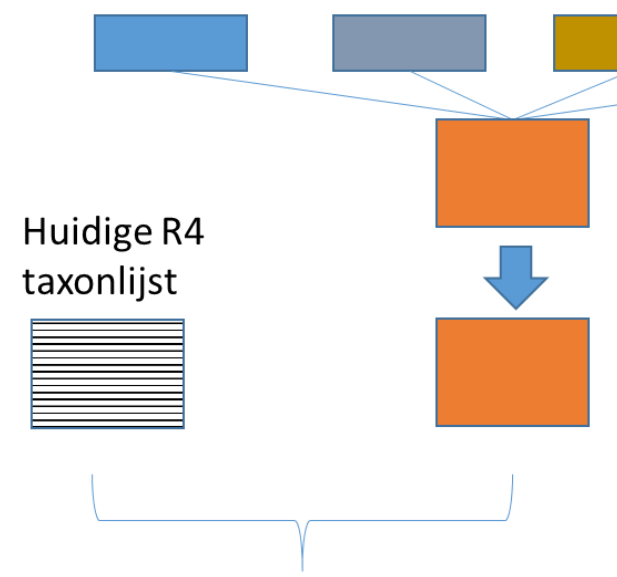

1 geaggregeerde dataset

Verschillen/overeenkomsten in KM, DP en DN taxa

Taxonomisch gestandaardiseerde dataset:

meest recente naamgeving, synoniemen, stadium

Doorvoeren aanpassingen

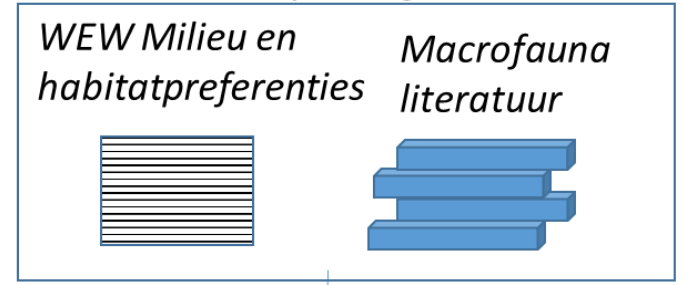

\section{Aangepast lijst met KM, DP en DN taxa}
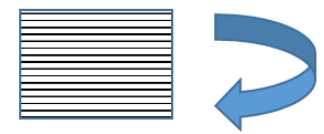

Feedback; aanpassingen

Figuur 2: Stappenplan herziening macrofaunamaatlat R4

\subsection{Vaststellen grenswaarden aangepaste maatlat}

Nadat de nieuwe indicatorlijst is opgesteld, is voor alle punten het percentage kenmerkende soorten (KM\%) berekend, opgedeeld naar regio en verhang. Dit is het aantal als kenmerkend aangeduide taxa gedeeld door het totaal aantal taxa. Dit percentage is belangrijk omdat dit voor een groot deel de uiteindelijke EKR score van een monster bepaalt. Het KM\% wordt per watertype geijkt aan een KMmax. Voor het watertype R4 bedraagt dit $26 \%$ in de huidige maatlat.

Voor de aangepaste soortenlijst moet de KMmax opnieuw worden vastgesteld. Om een beeld te krijgen van de KM\% van de aangepaste soortenlijst in de Nederlandse bovenlopen is per verhang-type (laag verhang en reliëfrijk) en beheergebied het maximale KM\% bepaald. Ook zijn het gemiddelde en het $95 \%$ percentiel bepaald om de spreiding in de KM\% per beheergebied in beeld te krijgen. Om te voorkomen dat onvolledige monsters invloed hebben op het eindresultaat, zijn alleen monsters met $2 / 3$ van het aantal taxonomische hoofdgroepen, 15 of meer taxa gebruikt. Tegelijkertijd heeft een aantal deelnemende waterschappen een lijst gemaakt van hun 'beste' (minst verstoorde) locaties. De aangeleverde locaties zijn vergeleken met de locaties waarbij uit de berekening de hoogste $\mathrm{KMmax}$ kwam bij wijze van controle of het $\mathrm{KM} \%$ een goed beeld geeft van de hoogste op dit moment aanwezige kwaliteit.

De definitieve KMmax is vastgesteld door het gemiddelde te nemen van de hoogste waarden van de drie beheergebieden met de hoogste KM\%-waarden. Er zijn verschillende beheergebieden genomen, immers moet de maatlat als landelijke beoordeling kunnen dienen en niet alleen een regionale beoordeling geven. Dit is apart gedaan voor de laag verhang en reliëfrijke beken. 
Nadat de nieuwe KMmax voor laag verhang en reliëfrijke beken is vastgesteld, zijn alle locaties met de nieuwe maatlat en de originele maatlat beoordeeld, zodat het effect van de aanpassingen duidelijk wordt.

\section{Resultaten}

\subsection{Aanpassingen indicatortaxalijst}

Er is een groot aantal wijzigingen in de indicatortaxalijst voor R4 doorgevoerd. In totaal is het aantal kenmerkende taxa uitgebreid met maar liefst 99 taxa (van 147 naar 246) en zijn er verschillende wijzigingen doorgevoerd in de positief dominante en negatief dominante taxa ( $P$ van 16 naar 31, N van 62 naar 37). De wijzigingen wisselen sterk in omvang per hoofdgroep (Tabel 2). De complete lijst inclusief de bijbehorende literatuurverwijzingen voor de indicaties is te vinden in Bijlage 1.

Er zijn ook soorten opgenomen die niet in de dataset aangetroffen zijn. Het gaat hierbij om circa een kwart van de taxa in de totale lijst. Dit zijn vooral zeer kritische/specialistische soorten die op dit moment in Nederland zeer zeldzaam of verdwenen zijn. Deze soorten moeten wel onderdeel zijn van de maatlat omdat als ze (weer) gevonden worden, deze door hun hoge indicatiewaarde wel mee moeten tellen. Het niet vinden van deze soorten heeft overigens geen effect op de EKR-score.

Tabel 2: Aanpassingen per taxonomische hoofdgroep. Afkortingen: $t$ : toegevoegd, a: afgevoerd, g: gehandhaafd, w: andere indicatie t.o.v. huidige maatlat.

\begin{tabular}{|c|c|c|c|c|c|c|c|c|c|c|c|c|}
\hline \multirow{3}{*}{ Hoofdgroep } & \multicolumn{12}{|c|}{ Indicatie (aantal taxa per hoofdgroep) } \\
\hline & \multicolumn{4}{|c|}{ Kenmerkend } & \multicolumn{4}{|c|}{ Dominant positief } & \multicolumn{4}{|c|}{ Dominant negatief } \\
\hline & $t$ & a & g & w & $\mathbf{t}$ & $\mathbf{a}$ & g & w & $\mathbf{t}$ & $\mathbf{a}$ & g & w \\
\hline Hirudinea (bloedzuigers) & 1 & & & & & & & & 1 & 3 & 3 & \\
\hline Oligochaeta (borstelwormen) & 3 & & 1 & & 1 & & & & & 14 & 6 & \\
\hline Tricladida (platwormen) & & & 3 & & & 1 & & & 1 & 1 & 1 & \\
\hline Hydracarina (watermijten) & 37 & & 9 & & 1 & 1 & & & & & & \\
\hline Amphipoda (vlokreeften) & & & & & & & 3 & & 1 & & & \\
\hline Decapoda (kreeften) & 1 & & & & & & & & & & & \\
\hline Isopoda (waterpissebedden) & & & & & & & & & 1 & 1 & & \\
\hline Diptera: Chironomidae (verdermuggen) & 30 & 3 & 17 & & 6 & 3 & 5 & & 1 & 4 & 7 & \\
\hline Diptera: Simuliidae (kriebelmuggen) & 1 & 1 & 6 & 1 & 2 & 2 & & & & & & \\
\hline Diptera: overig (andere vliegen en muggen) & 5 & 2 & 1 & & 2 & & & & 1 & & 2 & \\
\hline Coleoptera (kevers) & 17 & 8 & 20 & & 1 & 1 & & & & & & \\
\hline Ephemeroptera (haften) & 9 & 1 & 7 & & & & 1 & & & 2 & 1 & \\
\hline Heteroptera (wantsen) & 1 & 1 & 5 & & & & & & & & & \\
\hline Odonata (libellen) & 4 & 2 & 2 & & 1 & & & & & & & \\
\hline Plecoptera (steenvliegen) & & 1 & 8 & & 1 & & & & & & & \\
\hline Neuroptera (gaasvliegen) & & 1 & 1 & & & & & & & & & \\
\hline Megaloptera (slijkvliegen) & & & 1 & & & & & & & 1 & & \\
\hline Trichoptera (kokerjuffers) & 22 & 11 & 30 & 1 & 1 & & & 2 & & & & \\
\hline Bivalvia (tweekleppigen) & 1 & & & & 1 & & & & & & 1 & \\
\hline Gastropoda (slakken) & 1 & & & & & & & & & 4 & 10 & \\
\hline
\end{tabular}




\subsection{Vaststellen en toetsen grenswaarden aangepaste maatlat}

Tabel 3 geeft een overzicht van de KM\% voor de verschillende regio's en de bijbehorende beste beken op basis van de monstersamenstelling. Opvallend is het grote verschil tussen de gebieden. Duidelijk is dat reliëfrijke gebieden een hoger $\mathrm{KM} \%$ hebben. Het verschil tussen de gemiddelde $\mathrm{KM} \%$ en met name de $5 \%$ hoogste waarden en het maximum laat zien dat er maar weinig beken met een hoog KM\% zijn in de verschillende beheergebieden.

Tabel 3: Gemiddelde (Gem.), 95\% percentiel en maximale waarden (Max.) voor KM\% bepaald met aangepaste soortenlijst (Om te corrigeren voor onvolledige monsters zijn, alleen monsters met 2/3 van het aantal taxonomische hoofdgroepen, 15 of meer taxa, gebruikt).

g.g. geen gegevens.

\begin{tabular}{|c|c|c|c|c|c|c|c|}
\hline \multirow[b]{2}{*}{ Waterschap } & \multirow[b]{2}{*}{$\begin{array}{l}\text { Verhang- } \\
\text { type }\end{array}$} & \multicolumn{4}{|l|}{ KM\% } & \multirow[b]{2}{*}{$\mathbf{n}$} & \multirow{2}{*}{$\begin{array}{l}\text { Aangeduid } \\
\text { als 'goede } \\
\text { locatie' } \\
\text { door } \\
\text { beheerder }\end{array}$} \\
\hline & & Gem. & $95 \%$ percentiel & Max. & $\begin{array}{l}\text { Monster met } \\
\text { hoogste KM\% }\end{array}$ & & \\
\hline De Dommel & laagland & 7.4 & 19.6 & 30.5 & $\begin{array}{l}\text { Keersop, } \\
\text { benedenstrooms } \\
\text { Vlieterdijk } \\
\text { schaduwdeel } \\
\end{array}$ & 236 & ja \\
\hline Hunze en Aa's & laagland & 7.8 & 17.7 & 18.4 & $\begin{array}{l}\text { Anreper ruimsloot, } \\
\text { bostraject }\end{array}$ & 6 & g.g. \\
\hline Vallei en Veluwe & laagland & 5.9 & 17.5 & 36.7 & Koudebeek & 208 & g.g. \\
\hline Vechtstromen & laagland & 4.6 & 17.0 & 18.6 & $\begin{array}{l}\text { Schoolbeek, } \\
\text { Baardinksweg, } \\
\text { Twekkelo }\end{array}$ & 93 & g.g. \\
\hline Brabantse Delta & laagland & 5.7 & 15.6 & 22.2 & $\begin{array}{l}\text { Strijbeekse Beek, } \\
\text { Bovenstrooms } \\
\text { stuw } \\
\text { strijbeekseweg }\end{array}$ & 221 & ja \\
\hline Limburg & laagland & 4.8 & 11.4 & 15.2 & $\begin{array}{l}\text { Oude Graaf } \\
\text { achterste hout }\end{array}$ & 121 & g.g. \\
\hline Aa en Maas & laagland & 3.5 & 11.1 & 21.9 & $\begin{array}{l}\text { Esperloop Bakel, } \\
\text { Beekse peeldijk }\end{array}$ & 147 & ja \\
\hline Fryslân & laagland & 0.0 & 0.0 & 0.0 & n.v.t. & 6 & n.v.t. \\
\hline Rijn \& IJssel & reliëfrijk & 23.7 & 47.2 & 51.7 & Snijdersveerbeek & 43 & ja \\
\hline Vallei en Veluwe & reliëfrijk & 19.8 & 40.8 & 60.7 & $\begin{array}{l}\text { Geelmolenbeek } \\
\text { Vaassen }\end{array}$ & 172 & g.g. \\
\hline Vechtstromen & reliëfrijk & 18.9 & 40.3 & 61.1 & $\begin{array}{l}\text { Tankenberg-West } \\
\text { Bronbeek, } \\
\text { Tankenbergweg, } \\
\text { De Lutte }\end{array}$ & 127 & g.g. \\
\hline Limburg & reliëfrijk & 11.2 & 28.6 & 40.6 & $\begin{array}{l}\text { Pepinusbeek } \\
\text { Haeselaarbr. } \\
\text { Open }\end{array}$ & 137 & ja \\
\hline Rivierenland & reliëfrijk & 6.9 & 11.4 & 12.2 & $\begin{array}{l}\text { Groesbeek - Lage } \\
\text { Horst - } \\
\text { Drulsebeek }\end{array}$ & 5 & g.g. \\
\hline
\end{tabular}


Om de KMmax te bepalen, is het gemiddelde genomen van de drie hoogste waarden die zijn vastgesteld voor de verschillende beheergebieden; dit leidt tot een $\mathrm{KMmax}=30$ voor gebieden met een laag verhang en een $\mathrm{KMmax}=58$ voor reliëfrijke gebieden. Van een deel van de deelnemende waterschappen zijn inschattingen van de 'beste' (minst verstoorde) beken ontvangen; in alle gevallen kwam dit overeen met de berekende hoogste waarden.

\subsection{De maatlat}

De maatlat gaat uit van een standaard KRW-bemonstering. Op basis van het monster worden de waarden voor drie parameters berekend met behulp van de indicatortaxalijsten in Bijlage 1 (analoog aan de huidige R4-maatlat; Van der Molen et al., 2012):

- De parameter DN\%abun wordt berekend door de abundantie van de taxa die zowel in het monster als de lijsten negatief dominante indicatoren voorkomen om te zetten naar een abundantieklasse (Tabel 4) en te sommeren en vervolgens te delen door de som van alle abundantieklassen voor alle taxa.

- De parameter KM\%taxa wordt berekend door het aantal taxa dat zowel in het monster als de lijsten met kenmerkende taxa voorkomt te delen door het totaal aantal taxa in het monster.

- De parameter KM\%abun + DP\%abun wordt berekend door de abundanties van taxa die zowel in het monster als de lijsten kenmerkende taxa of positief dominante indicatoren voorkomen om te zetten naar een abundantieklasse en te sommeren en vervolgens te delen door de som van alle abundantieklassen voor alle taxa.

Tabel 4: Omrekening van abundantie naar abundantieklasse voor gebruik in de EKRformule.

\begin{tabular}{|l|l|}
\hline Abundantie & Klasse \\
\hline 0 & 0 \\
\hline$>0,<1.5$ & 1 \\
\hline$\geq 1.5,<4.5$ & 2 \\
\hline$\geq 4.5,<12.5$ & 3 \\
\hline$\geq 12.5,<33.5$ & 4 \\
\hline$\geq 33.5,<90.5$ & 5 \\
\hline$\geq 90.5,<244.5$ & 6 \\
\hline$\geq 244.5,<665.5$ & 7 \\
\hline$\geq 665.5,<1808.5$ & 8 \\
\hline$\geq 1808.5$ & 9 \\
\hline
\end{tabular}

Als kwaliteitsijkpunt wordt de hierboven bepaalde KMmax gebruikt, het percentage kenmerkende taxa dat onder referentieomstandigheden kan worden verwacht in een monster. De term $\mathrm{KM}_{\operatorname{tax}} \% / \mathrm{KMmax}$ in de EKR-formule is gelimiteerd, als $\mathrm{KM}_{\operatorname{tax}} \% / \mathrm{KMmax}>1$ (oftewel een hoger aantal kenmerkende taxa dan onder referentieomstandigheden) dan wordt in de formule gerekend met 1.

Met de scores van bovenstaande parameters wordt vervolgens in een formule de EKR uitgerekend. Deze formule is gelijk aan de oorspronkelijke R4-maatlat, alleen wordt er een verschillende KMmax gebruikt voor R4 beken in vlakke gebieden en in reliëfrijke gebieden:

$E K R=\frac{200 *\left(K M \%_{\text {taxa }} / \mathrm{KMmax}\right)+2 *\left(100-\mathrm{DN} \%_{\text {abun }}\right)+\left(D P \%_{\text {abun }}+K M \%_{\text {abun }}\right)}{500}$ 
KMmax: Voor R4 beken in vlakke gebieden wordt $\mathrm{KMmax}=30$ gehanteerd, voor beken in reliëfrijke gebieden $\mathrm{KMmax}=58$. De keuze voor laag verhang of reliëfrijk is af te leiden uit figuur 1 en tabel 1.

Wanneer de monsters uit 2007-2017 worden doorgerekend met de aangepaste soortenlijsten voor laagland en reliëfrijke gebieden valt op dat vooral de vlakke gebieden met de nieuwe maatlat hoger scoren ten opzichte van de oorspronkelijke maatlat; met name de monsters met een betere kwaliteit (Figuur 4).
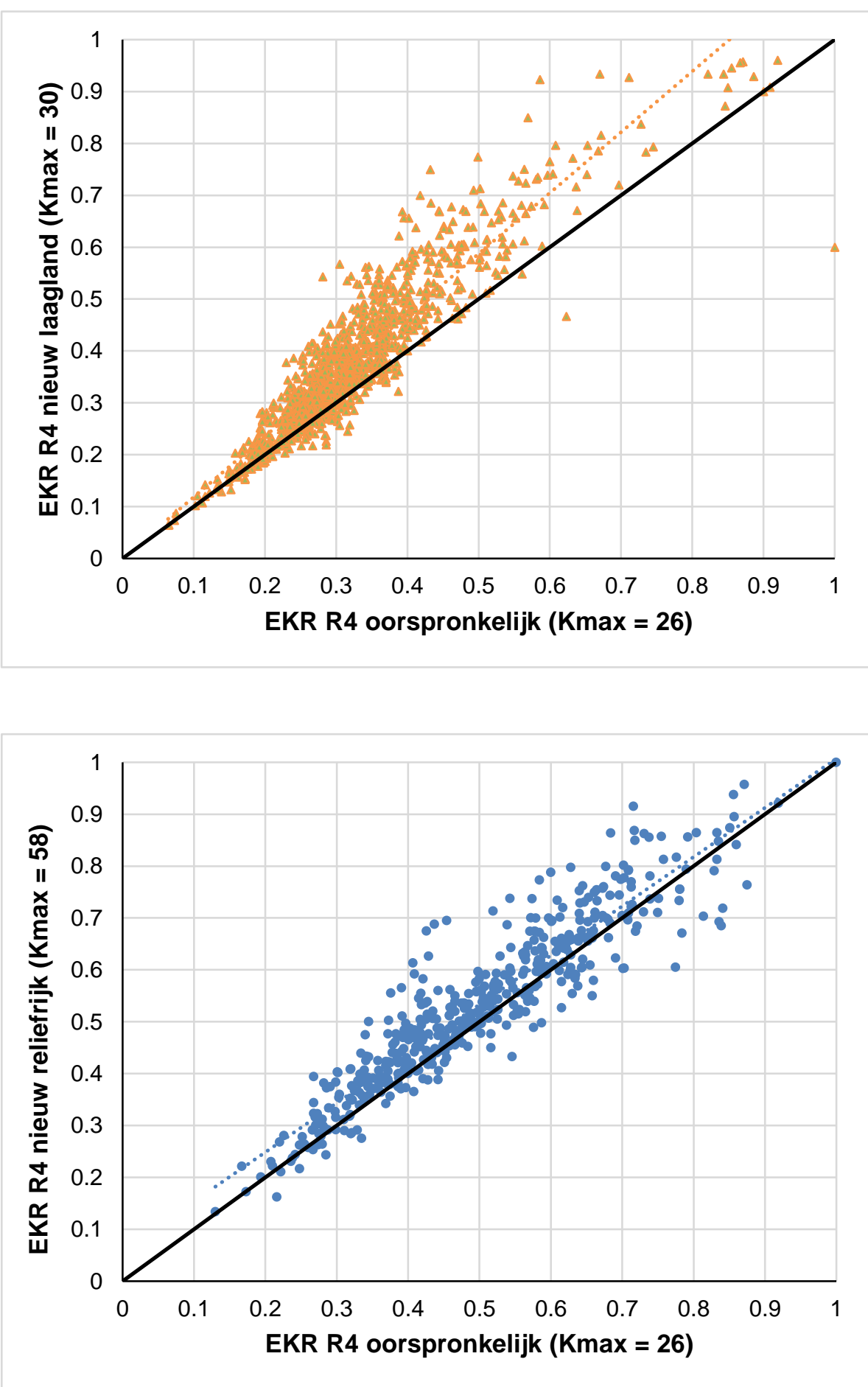

Figuur 4: Vergelijking tussen de nieuwe maatlat en de oorspronkelijke maatlatscore voor gebieden met relatief weinig verhang (laagland) en reliëfrijke gebieden. De zwarte lijn geeft de theoretische gelijke score aan, de stippellijn de lineaire trendlijn op basis van de data. 
Met de huidige maatlat werd $2,7 \%$ van het totaal aantal monsters uit gebieden met een laag verhang als goed (EKR $\geq 0,6 ; n$ totaal $=1061$ monsters) beoordeeld, met de aangepaste maatlat stijgt dit aantal naar $8,5 \%$. Voor reliëfrijke gebieden geldt dat de beoordeling vergelijkbaar blijft, ondanks de sterke verhoging van de KMmax. Met de huidige maatlat werd $25,9 \%$ van het totaal aantal monsters uit de reliëfrijke gebieden als goed (EKR $\geq 0,6 ; n$ totaal $=460$ monsters) beoordeeld, met de aangepaste maatlat stijgt dit aantal naar 31,4\%. De spreiding van de data over de kwaliteitsgradiënt is voor de laagland R4-beken geclusterd in de zone met een lagere kwaliteit (de meeste monsters krijgen een relatief lage EKR), voor de reliëfrijke gebieden is dit meer gelijk verdeeld. Wel komen in beide gevallen op de maatlat voor de natuurlijke referentie monsters met zowel slechte als zeer goede beoordelingen voor.

\section{Discussie en aanbevelingen}

\section{KMmax}

Voor sommige andere maatlatten is de KMmax bepaald door deze op basis van expert judgement te ijken. Hierbij werd gekeken naar welke monsters een EKR score van 0,6 EKR zouden moeten halen op basis van een inschatting van de kwaliteit en hierop werd vervolgens de KMmax op bijgestuurd (med. Jeroen van Mil). Deze aanpak wijkt af van de hier gehanteerde methode waarbij de hoogste berekende $\mathrm{KM} \%$ van beken in verschillende regio's zijn gebruikt en getoetst is of de beken waarvoor de hoogst berekende waarden werden gevonden ook door de regionale waterbeheerders werden beschouwd als horende bij de 'beste' beken. Er is voor deze aanpak gekozen omdat zo de beoordeling wordt afgeleid van de beste bovenlopen die op dit moment in Nederland te vinden zijn. Omdat dit veelal al beken in natuurgebieden zijn met een relatief lager aantal stressoren dat invloed uitoefent op het systeem kan dit worden beschouwd als het best haalbare voor de Nederlandse situatie. Consequentie van de aanpak is dat de betreffende monsters een EKR zeer goed krijgen (in theorie EKR = 1 wanneer er geen dominant negatieve taxa zouden worden aangetroffen, maar dit komt in de praktijk niet voor).

\section{Typering/naamgeving subtypen}

Er wordt voor de herziene R4 maatlat onderscheid gemaakt tussen twee subtypen voor R4. Door de werkgroep doelstellingen is de volgende naamgeving vastgesteld:

- subtype R4a voor een levensgemeenschap in bovenlopen met een relatief laag verhang: permanente langzaam stromende laaglandbeek op zand.

- subtype R4b voor levensgemeenschappen in bovenlopen in reliëfrijke gebieden: permanente langzaam stromende heuvelland bovenloop op zand.

Tabel 1 in combinatie met de indeling in hydrobiologische districten (Mol 1986) geeft een indicatie van welk subtype gehanteerd moet worden. Bij het toepassen van de maatlat is het dus belangrijk beek-specifiek te kijken naar 1.) het verhang en de 2.) de stroomsnelheidsrange van de beek, en op basis hiervan tot keuze van het subtype te komen.

\section{Samenhang met andere typen}

De bovenlopen van laaglandbeken zijn onder te verdelen langs een gradient van een zeer laag verhang en zeer lage stroomsnelheid (doorstroommoeras) naar snelstromende bovenlopen (zie ranges in Tabel 1). In deze herziening is dit laatste type - KRW beektype R13, snelstromende bovenloop op zand - buiten beschouwing gelaten. Volgens de KRW typologie vraagt dit type naast een hoog verhang een stroomsnelheid van boven de $50 \mathrm{~cm} / \mathrm{s}$, wat relatief hoog is voor een Nederlandse laaglandbeek (Verdonschot 2000). Het is dan ook niet verwonderlijk dat slechts aan een beperkt aantal bovenlopen dit type is toegekend, grotendeels in het zuidelijk deel van Limburg. Het is daarom geen verrassing dat dit type qua levensgemeenschap veel overlap heeft met het KRW-type snelstromende bovenlopen op 
kalkhoudende bodem (bovenlopen in het Zuid Limburgse heuvelland met een mergelondergrond). De maatlat bevat veel soorten van de levensgemeenschappen die optimaal ontwikkeld zijn in de midden/centraal Europese middelgebergten (zoals Eifel, Ardennen). Tegelijkertijd bevat R13 soorten van de laaglandbeeklevensgemeenschappen en treedt er overlap op met de huidige en de herziene R4 maatlat. De overeenkomsten en verschillen tussen de herziene R4 maatlat subtype R4b en $\mathrm{R} 13$ zouden nog nader bekeken moeten worden; mogelijk is R4b zelfs een alternatief voor R13, maar dan met een grotere landelijke representativiteit.

Leidt een groter aantal indicatorsoorten tot lagere EKR scores?

In de aangepaste soortenlijst is het aantal indicatoren hoger dan in de huidige lijst. Een langere lijst met indicatoren leidt niet tot lagere EKR scores, omdat de KMmax niet geijkt is op indicatoren die niet worden gevonden, maar uitsluitend op de taxa die wel worden aangetroffen in een monster (wel op basis van de beste locaties). Een hoger aantal kenmerkende indicatoren leidt dus in beginsel tot een hogere EKR score. Wat wel sterk doorwerkt op de EKR is het aantal aangetroffen soorten die geen indicatieve taxa betreffen. Worden er veel niet scorende taxa gevonden ten opzichte van het aantal wel scorende taxa, dan daalt de EKR. Dit is ook de reden dat er een groot verschil is tussen de KMmax van laag verhang bovenlopen en de KMmax van bovenlopen in reliefrijke gebieden. De eerste groep bevat door de lagere stroomsnelheid ook relatief veel niet scorende stilstaand water soorten, waardoor het $\mathrm{KM} \%$ automatisch daalt. Het aantal kenmerkende taxa gedeeld door het totaal aantal taxa in het monster is dan immers lager.

Intercalibratie

Intercalibratie wordt niet nodig geacht, omdat wijzigingen slechts van toepassing zijn op één type en alleen een aanpassingen van soortenlijst en de daarvan afgeleide constanten betreffen.

\section{Herziening tekst maatlatdocument macrofauna}

Hieronder worden tekstvoorstellen gegeven voor aanpassingen in het maatlatdocument (Van der Molen et al. 2012)

\subsection{Globale referentiebeschrijving, subkop Macrofauna (p. 136)}

De macrofauna leeft met name in of op het sediment (zand, detritus) of op harde substraten (grind, hout). Steenvliegen, kokerjuffers, haften, waterkevers, vliegen en muggen zijn een selectie van de belangrijke groepen. In voedselarme bovenlopen is de macrofauna matig divers en heeft lage aantallen individuen. Opvallend is het sporadisch voorkomen of ontbreken van veel soorten haften, platwormen, slakken en kreeftachtigen. De meeste soorten leven op het sediment (de steenvlieg Leuctra nigra en de kriebelmug Simulium aureum) of in het sediment (de vedermug Heterotanytarsus apicalis, de libel Cordulegaster boltonii en de slijkvlieg Sialis fuliginosa). Het betreft vooral heterotrofe vergaarders en knippers. Belangrijke groepen zijn vedermuggen (Corynoneura lobata, Micropsectra pallidula en Stempellinella edwardsi), steenvliegen (Leuctra nigra en Nemurella pictetii) en kevers (Hydroporus discretus, Hydraena riparia). In de wat voedselrijkere bovenlopen komt een meer diverse macrofaunagemeenschap voor. De meeste soorten leven op vaste substraten (de kriebelmug Simulium cryophilum, de kevers Limnebius truncatellus en Elmis aenea) en in mindere mate in het sediment (de vedermuggen Brillia bifida en Chaetocladius gr. vitellinus). Veel soorten zijn rheobiont (de kokerjuffers Tinodes assimilis en Potamophylax cingulatus), rheofiel (de kevers Helophorus arvenicus, Platambus maculatus, Agabus didymus, Nebrioporus elegans) en koud stenotherm. Het betreft detriti-herbivoren, carnivoren en omnivoren. Belangrijke groepen zijn steenvliegen (Amphinemura standfussi), kokerjuffers 
(Micropterna sequax), haften (Baetis niger, Procloeon bifidum, Centrophilum luteolum), keeftachtigen (Gammarus fossarum, G. pulex), watermijten (Sperchon squamosus en Sperchon turgidus), kevers (Limnius volckmari) en libellen (Calopteryx virgo).

12.3 Macrofauna: abundantie en soortensamenstelling (p. 137)

Met de scores voor het relatief aandeel negatief dominante indicatoren (DN\%) en de kenmerkende en positief dominante indicatoren (KM\% + DP\%) en het percentage kenmerkende taxa (KM\%) wordt in een formule de EKR uitgerekend zoals in hoofdstuk 2 is uiteengezet. Voor indicatoren op een hoger taxonomisch niveau (bijv. genus, familie) worden alle onderliggende taxa beschouwd als behorend tot deze indicator. De abundanties worden eerst opgeteld voor aangetroffen soorten die behoren tot hetzelfde indicatortaxon en vervolgens worden abundanties omgezet naar klassen. De lijst met indicatorsoorten is opgenomen in bijlage (X). Bij dit watertype worden twee subtypen met een eigen KMmax onderscheiden. Voor vlakke gebieden (R4a, permanente langzaam stromende laaglandbeek op zand) geldt een KMmax $=30$, voor reliëfrijke gebieden ( $R 4 b$, permanente langzaam stromende heuvelland bovenloop op zand) een KMmax $=58$. Het onderscheid tussen de subtypen wordt gemaakt op basis van verhang en stroomsnelheidsrange, waarbij geldt verhang $0,5-1 \mathrm{~m} / \mathrm{km}$ voor R4a en $>1 \mathrm{~m} / \mathrm{km}$ voor R4b met stroomsnelheden van $10-50 \mathrm{~cm} / \mathrm{s}$ voor R4a en $30-80 \mathrm{~cm} / \mathrm{s}$ voor R4b.

\section{Literatuur}

Mol, A.W.M. (1986) Hydrobiologische districten in Nederland. De Levende Natuur 87: 79-86.

van der Molen, D.T., Pot, R., Evers, C.H.M., Nieuwerburgh, L.L.J. van, (2012) Referenties en maatlatten voor natuurlijke watertypen voor de Kaderrichtlijn Water 2015-2021. Rapport 201231. STOWA, Amersfoort.

Verdonschot, P.F.M. (2000) Natuurlijke levensgemeenschappen van de Nederlandse binnenwateren deel 2, Beken. Achtergronddocument bij het 'Handboek Natuurdoeltypen in Nederland'. Rapport EC-LNV nr. AS-02. Expertisecentrum LNV, Wageningen.

Verdonschot, R.C.M., Verdonschot, P.F.M. (2017) Relatie KRW-doelen en macrofauna in beken in Noord-Brabant. Wageningen Environmental Research, Wageningen UR, Wageningen.

Verdonschot, R., Runhaar, H., Buijse, T., Bijkerk, R., Verdonschot, P. (2016) Doorstroommoerassen en moerasbeken. Typebeschrijvingen en ontwikkeling maatlatten voor de biologische kwaliteitselementen. Notitie Zoetwatersystemen, Alterra Wageningen UR, Wageningen.

Verberk, W.C.E.P., Verdonschot, P.F.M., van Haaren, T., van Maanen, B. (2012) Milieu- en habitatpreferenties van Nederlandse zoetwatermacrofauna. WEW Themanummer 23, Van de Garde-Jémé, Eindhoven. 


\section{Bijlage 1: Indicatorlijst}

Huidige (R4 origineel) en herziene R4 soortenlijst voor langzaam stromende bovenlopen op zand. Indicatiewaarden $1=\mathrm{KM}, 2=\mathrm{DP}, 3=\mathrm{DN}$. Voor een indicator van een hoger taxonomisch niveau wordt met hiertoe behorende taxa gerekend als zijnde deze indicator. $\mathrm{x}=\mathrm{geen}$ literatuur gevonden, inschatting.

\begin{tabular}{|c|c|c|c|c|c|}
\hline Taxonnaam TWN & $\begin{array}{l}\text { Hoofdgroep } \\
\text { TWN }\end{array}$ & Familie & \begin{tabular}{|l} 
R4 \\
herzien
\end{tabular} & $\begin{array}{l}\text { R4 } \\
\text { origineel }\end{array}$ & Referentie(s) \\
\hline Erpobdella nigricollis & APHIR & Erpobdellidae & 3 & & van Haaren et al. 2004 \\
\hline Erpobdella octoculata & APHIR & Erpobdellidae & 3 & 3 & van Haaren et al. 2004 \\
\hline Erpobdella testacea & APHIR & Erpobdellidae & 3 & 3 & van Haaren et al. 2004 \\
\hline Erpobdella vilnensis & APHIR & Erpobdellidae & 1 & & van Haaren et al. 2004 \\
\hline Alboglossiphonia heteroclita & APHIR & Glossiphoniidae & & 3 & van Haaren et al. 2004 \\
\hline Glossiphonia complanata & APHIR & Glossiphoniidae & & 3 & van Haaren et al. 2004 \\
\hline Helobdella stagnalis & APHIR & Glossiphoniidae & 3 & 3 & van Haaren et al. 2004 \\
\hline Hemiclepsis marginata & APHIR & Glossiphoniidae & & 3 & van Haaren et al. 2004 \\
\hline Haplotaxis gordioides & APOLI & Haplotaxidae & 1 & & van Haaren \& Soors 2013 \\
\hline Stylodrilus heringianus & APOLI & Lumbriculidae & 2 & & van Haaren \& Soors 2013 \\
\hline Chaetogaster diaphanus & APOLI & Naididae & & 3 & van Haaren \& Soors 2013 \\
\hline Chaetogaster diastrophus & APOLI & Naididae & & 3 & van Haaren \& Soors 2013 \\
\hline Chaetogaster limnaei & APOLI & Naididae & & 3 & van Haaren \& Soors 2013 \\
\hline Dero digitata & APOLI & Naididae & 3 & 3 & van Haaren \& Soors 2013 \\
\hline Nais alpina & APOLI & Naididae & 1 & 1 & van Haaren \& Soors 2013 \\
\hline Nais communis & APOLI & Naididae & 3 & 3 & van Haaren \& Soors 2013 \\
\hline Nais elinguis & APOLI & Naididae & 3 & 3 & van Haaren \& Soors 2013 \\
\hline Nais pseudobtusa & APOLI & Naididae & & 3 & van Haaren \& Soors 2013 \\
\hline Ophidonais serpentina & APOLI & Naididae & 3 & 3 & van Haaren \& Soors 2013 \\
\hline Specaria josinae & APOLI & Naididae & 1 & & van Haaren \& Soors 2013 \\
\hline Stylaria lacustris & APOLI & Naididae & 3 & 3 & van Haaren \& Soors 2013 \\
\hline
\end{tabular}




\begin{tabular}{|c|c|c|c|c|c|}
\hline Taxonnaam TWN & $\begin{array}{l}\text { Hoofdgroep } \\
\text { TWN }\end{array}$ & Familie & $\begin{array}{l}\text { R4 } \\
\text { herzien }\end{array}$ & $\begin{array}{l}\text { R4 } \\
\text { origineel }\end{array}$ & Referentie(s) \\
\hline Tubificidae & APOLI & Tubificidae & 3 & 3 & van Haaren \& Soors 2013 \\
\hline Limnodrilus & APOLI & Tubificidae & & 3 & $x$ \\
\hline Potamothrix & APOLI & Tubificidae & & 3 & $\mathrm{x}$ \\
\hline Aulodrilus limnobius & APOLI & Tubificidae & & 3 & van Haaren \& Soors 2013 \\
\hline Embolocephalus velutinus & APOLI & Tubificidae & 1 & & van Haaren \& Soors 2013 \\
\hline Limnodrilus claparedianus & APOLI & Tubificidae & & 3 & $\mathrm{x}$ \\
\hline Limnodrilus hoffmeisteri & APOLI & Tubificidae & & 3 & $x$ \\
\hline Limnodrilus udekemianus & APOLI & Tubificidae & & 3 & $x$ \\
\hline Potamothrix hammoniensis & APOLI & Tubificidae & & 3 & $\mathrm{x}$ \\
\hline Potamothrix moldaviensis & APOLI & Tubificidae & & 3 & $x$ \\
\hline Psammoryctides barbatus & APOLI & Tubificidae & & 3 & $\mathrm{x}$ \\
\hline Rhyacodrilus coccineus & APOLI & Tubificidae & & 3 & $\mathrm{x}$ \\
\hline Dugesia gonocephala & APTUR & Dugesiidae & 1 & 1 & Den Hartog 1962 \\
\hline Schmidtea lugubris & APTUR & Dugesiidae & 3 & 3 & Reynoldson \& Young 2000 \\
\hline Schmidtea polychroa & APTUR & Dugesiidae & & 2 & Reynoldson \& Young 2000 \\
\hline Crenobia alpina & APTUR & Planariidae & 1 & 1 & Reynoldson \& Young 2000 \\
\hline Polycelis felina & APTUR & Planariidae & 1 & 1 & Reynoldson \& Young 2000 \\
\hline Polycelis nigra/tenuis & APTUR & Planariidae & 3 & & Den Hartog 1962 \\
\hline Polycelis tenuis & APTUR & Planariidae & & 3 & $x$ \\
\hline Bandakia concreta & $\mathrm{ARACH}$ & Anisitsiellidae & 1 & & Smit \& van der Hammen 2000 \\
\hline Arrenurus cylindratus & ARACH & Arrenuridae & 1 & 1 & Smit \& van der Hammen 2000 \\
\hline Arrenurus octagonus & ARACH & Arrenuridae & 1 & & Smit \& van der Hammen 2000 \\
\hline Arrenurus zachariasi & ARACH & Arrenuridae & 1 & & Smit \& van der Hammen 2000 \\
\hline Aturus fontinalis & ARACH & Aturidae & 1 & & Gerecke et al. 2016 \\
\hline Ljania bipapillata & ARACH & Aturidae & 1 & & Gerecke et al. 2016 \\
\hline Hydrodroma torrenticola & ARACH & Hydrodromidae & 1 & & Di Sabatino et al. 2010 \\
\hline Protzia eximia & ARACH & Hydryphantidae & 1 & 1 & Di Sabatino et al. 2010 \\
\hline Tartarothyas romanica & ARACH & Hydryphantidae & 1 & & Di Sabatino et al. 2010 \\
\hline
\end{tabular}




\begin{tabular}{|c|c|c|c|c|c|}
\hline Taxonnaam TWN & $\begin{array}{l}\text { Hoofdgroep } \\
\text { TWN }\end{array}$ & Familie & $\begin{array}{l}\text { R4 } \\
\text { herzien }\end{array}$ & $\begin{array}{l}\mathbf{R 4} \\
\text { origineel }\end{array}$ & Referentie(s) \\
\hline Atractides distans & ARACH & Hygrobatidae & 1 & & Gerecke et al. 2016 \\
\hline Atractides nodipalpis [1] & ARACH & Hygrobatidae & 1 & & Gerecke et al. 2016 \\
\hline Atractides tener & ARACH & Hygrobatidae & 1 & & Gerecke et al. 2016 \\
\hline Hygrobates longiporus & ARACH & Hygrobatidae & 1 & & Smit \& van der Hammen 2000 \\
\hline Hygrobates nigromaculatus [1] & ARACH & Hygrobatidae & & 2 & Gerecke et al. 2016 \\
\hline Hygrobates setosus & ARACH & Hygrobatidae & 2 & & Gerecke et al. 2016 \\
\hline Hygrobates trigonicus & ARACH & Hygrobatidae & 1 & & Gerecke et al. 2016 \\
\hline Lebertia bracteata & ARACH & Lebertiidae & 1 & 1 & Di Sabatino et al. 2010 \\
\hline Lebertia cognata & ARACH & Lebertiidae & 1 & & Di Sabatino et al. 2010 \\
\hline Lebertia dubia & ARACH & Lebertiidae & 1 & & Di Sabatino et al. 2010 \\
\hline Lebertia fimbriata & ARACH & Lebertiidae & 1 & & Di Sabatino et al. 2010 \\
\hline Lebertia glabra & ARACH & Lebertiidae & 1 & & Di Sabatino et al. 2010 \\
\hline Lebertia insignis & ARACH & Lebertiidae & 1 & 1 & Di Sabatino et al. 2010 \\
\hline Lebertia minutipalpis & ARACH & Lebertiidae & 1 & 1 & Di Sabatino et al. 2010 \\
\hline Lebertia natans & $\mathrm{ARACH}$ & Lebertiidae & 1 & & Di Sabatino et al. 2010 \\
\hline Lebertia porosa & ARACH & Lebertiidae & 1 & & Di Sabatino et al. 2010 \\
\hline Lebertia rivulorum & ARACH & Lebertiidae & 1 & & Di Sabatino et al. 2010 \\
\hline Lebertia stigmatifera & $\mathrm{ARACH}$ & Lebertiidae & 1 & & Di Sabatino et al. 2010 \\
\hline Mideopsis crassipes & $\mathrm{ARACH}$ & Mideopsidae & 1 & & Gerecke et al. 2016 \\
\hline Mideopsis roztoczensis & ARACH & Mideopsidae & 1 & & Gerecke et al. 2016 \\
\hline Oxus ovalis & $\mathrm{ARACH}$ & Oxidae & 1 & & Di Sabatino et al. 2010 \\
\hline Oxus setosus & ARACH & Oxidae & 1 & & Smit \& van der Hammen 2000 \\
\hline Forelia liliacea & ARACH & Pionidae & 1 & & Smit \& van der Hammen 2000 \\
\hline Forelia longipalpis & ARACH & Pionidae & 1 & & Smit \& van der Hammen 2000 \\
\hline Nautarachna crassa & ARACH & Pionidae & 1 & & Gerecke et al. 2016 \\
\hline Sperchon clupeifer & ARACH & Sperchontidae & 1 & & Di Sabatino et al. 2010 \\
\hline Sperchon compactilis & ARACH & Sperchontidae & 1 & & Di Sabatino et al. 2010 \\
\hline Sperchon glandulosus & ARACH & Sperchontidae & 1 & 1 & Di Sabatino et al. 2010 \\
\hline
\end{tabular}




\begin{tabular}{|c|c|c|c|c|c|}
\hline Taxonnaam TWN & $\begin{array}{l}\text { Hoofdgroep } \\
\text { TWN }\end{array}$ & Familie & $\begin{array}{l}\text { R4 } \\
\text { herzien }\end{array}$ & $\begin{array}{l}\mathrm{R4} \\
\text { origineel }\end{array}$ & Referentie(s) \\
\hline Sperchon longissimus & $\mathrm{ARACH}$ & Sperchontidae & 1 & & Smit \& van der Hammen 2000 \\
\hline Sperchon setiger [1] & $\mathrm{ARACH}$ & Sperchontidae & 1 & 1 & Di Sabatino et al. 2010 \\
\hline Sperchon squamosus & $\mathrm{ARACH}$ & Sperchontidae & 1 & 1 & Di Sabatino et al. 2010 \\
\hline Sperchon thienemanni & $\mathrm{ARACH}$ & Sperchontidae & 1 & & Di Sabatino et al. 2010 \\
\hline Sperchon turgidus & ARACH & Sperchontidae & 1 & & Di Sabatino et al. 2010 \\
\hline Sperchon vaginosus & $\mathrm{ARACH}$ & Sperchontidae & 1 & & Di Sabatino et al. 2010 \\
\hline Sperchonopsis verrucosa & ARACH & Sperchontidae & 1 & & Di Sabatino et al. 2010 \\
\hline Teutonia cometes & $\mathrm{ARACH}$ & Teutoniidae & 1 & & Smit \& van der Hammen 2000 \\
\hline Torrenticola amplexa & $\mathrm{ARACH}$ & Torrenticolidae & 1 & & Di Sabatino et al. 2010 \\
\hline Neumania imitata & $\mathrm{ARACH}$ & Unionicolidae & 1 & & Gerecke et al. 2016 \\
\hline Wettina podagrica & ARACH & Wettinidae & 1 & 1 & Gerecke et al. 2016 \\
\hline Crangonyx pseudogracilis & CRAMP & Crangonyctidae & 3 & & $x$ \\
\hline Gammarus fossarum & CRAMP & Gammaridae & 2 & 2 & $\mathrm{x}$ \\
\hline Gammarus pulex & CRAMP & Gammaridae & 2 & 2 & $\mathrm{x}$ \\
\hline Gammarus roeseli & CRAMP & Gammaridae & 2 & 2 & $x$ \\
\hline Astacus astacus & CRDEC & Astacidae & 1 & & $x$ \\
\hline Asellidae & CRISO & Asellidae & 3 & & $x$ \\
\hline Asellus aquaticus & CRISO & Asellidae & & 3 & $x$ \\
\hline Chironomus & IDCHI & Chironomini & 3 & 3 & Moller Pillot 2009 \\
\hline Cladopelma goetghebueri gr. & IDCHI & Chironomini & & 3 & Moller Pillot 2009 \\
\hline Dicrotendipes nervosus & IDCHI & Chironomini & 3 & 3 & Moller Pillot 2009 \\
\hline Glyptotendipes & IDCHI & Chironomini & 3 & 3 & Moller Pillot 2009 \\
\hline Microtendipes chloris agg. & $\mathrm{IDCHI}$ & Chironomini & & 3 & Moller Pillot 2009 \\
\hline Paracladopelma camptolabis & IDCHI & Chironomini & 1 & 1 & Moller Pillot 2013 \\
\hline Paracladopelma nigritulum & IDCHI & Chironomini & 1 & & Moller Pillot 2013 \\
\hline Paratendipes albimanus & $\mathrm{IDCHI}$ & Chironomini & 2 & & Moller Pillot 2009 \\
\hline Phaenopsectra & IDCHI & Chironomini & & 3 & Moller Pillot 2009 \\
\hline Polypedilum albicorne & IDCHI & Chironomini & 1 & & Moller Pillot 2009 \\
\hline
\end{tabular}




\begin{tabular}{|c|c|c|c|c|c|}
\hline Taxonnaam TWN & $\begin{array}{l}\text { Hoofdgroep } \\
\text { TWN }\end{array}$ & Familie & $\begin{array}{l}\text { R4 } \\
\text { herzien }\end{array}$ & $\begin{array}{l}\mathrm{R4} \\
\text { origineel }\end{array}$ & Referentie(s) \\
\hline Polypedilum convictum & IDCHI & Chironomini & 1 & & Moller Pillot 2009 \\
\hline Polypedilum laetum & IDCHI & Chironomini & 1 & & Moller Pillot 2009 \\
\hline Polypedilum nubeculosum & IDCHI & Chironomini & 3 & 3 & Moller Pillot 2009 \\
\hline Polypedilum pedestre & IDCHI & Chironomini & 1 & 1 & Moller Pillot 2009 \\
\hline Polypedilum scalaenum & IDCHI & Chironomini & 2 & & Moller Pillot 2009 \\
\hline Stictochironomus maculipennis & IDCHI & Chironomini & 1 & & Moller Pillot 2009 \\
\hline Diamesa insignipes & IDCHI & Diamesinae & 1 & & Moller Pillot 2013 \\
\hline Brillia bifida & IDCHI & Orthocladiinae & 1 & 1 & Moller Pillot 2013 \\
\hline Brillia longifurca & IDCHI & Orthocladiinae & 1 & & Moller Pillot 2013 \\
\hline Chaetocladius femineus & IDCHI & Orthocladiinae & 1 & 1 & Moller Pillot 2013 \\
\hline Chaetocladius laminatus & IDCHI & Orthocladiinae & 1 & & Moller Pillot 2013 \\
\hline Chaetocladius melaleucus & IDCHI & Orthocladiinae & 1 & 1 & Moller Pillot 2013 \\
\hline Chaetocladius vitellinus gr. & IDCHI & Orthocladiinae & 1 & 1 & Moller Pillot 2013 \\
\hline Corynoneura coronata & IDCHI & Orthocladiinae & 1 & & Moller Pillot 2013 \\
\hline Corynoneura lobata agg. & IDCHI & Orthocladiinae & 1 & 1 & Moller Pillot 2013 \\
\hline Cricotopus bicinctus & IDCHI & Orthocladiinae & 3 & & Moller Pillot 2013 \\
\hline Cricotopus sylvestris gr. & IDCHI & Orthocladiinae & 3 & 3 & Moller Pillot 2013 \\
\hline Cricotopus tibialis & IDCHI & Orthocladiinae & 1 & & Moller Pillot 2013 \\
\hline Epoicocladius ephemerae & IDCHI & Orthocladiinae & 1 & 1 & Moller Pillot 2013 \\
\hline Eukiefferiella brevicalcar & IDCHI & Orthocladiinae & 1 & 1 & Moller Pillot 2013 \\
\hline Eukiefferiella claripennis & IDCHI & Orthocladiinae & & 1 & Moller Pillot 2013 \\
\hline Eukiefferiella gracei & IDCHI & Orthocladiinae & 1 & & Moller Pillot 2013 \\
\hline Heleniella ornaticollis & IDCHI & Orthocladiinae & 1 & & Moller Pillot 2013 \\
\hline Heterotanytarsus apicalis & IDCHI & Orthocladiinae & 1 & 1 & Moller Pillot 2013 \\
\hline Heterotrissocladius marcidus & IDCHI & Orthocladiinae & 1 & 1 & Moller Pillot 2013 \\
\hline Hydrobaenus pilipes & IDCHI & Orthocladiinae & 1 & 1 & Moller Pillot 2013 \\
\hline Nanocladius rectinervis & IDCHI & Orthocladiinae & 1 & 1 & Moller Pillot 2013 \\
\hline Orthocladius (Euorthocladius) & IDCHI & Orthocladiinae & 1 & & Moller Pillot 2013 \\
\hline
\end{tabular}




\begin{tabular}{|c|c|c|c|c|c|}
\hline Taxonnaam TWN & $\begin{array}{l}\text { Hoofdgroep } \\
\text { TWN }\end{array}$ & Familie & $\begin{array}{l}\text { R4 } \\
\text { herzien }\end{array}$ & $\begin{array}{l}\mathrm{R4} \\
\text { origineel }\end{array}$ & Referentie(s) \\
\hline Orthocladius frigidus & IDCHI & Orthocladiinae & 1 & & Moller Pillot 2013 \\
\hline Orthocladius lignicola & IDCHI & Orthocladiinae & 1 & & Moller Pillot 2013 \\
\hline Orthocladius oblidens & IDCHI & Orthocladiinae & 1 & & Moller Pillot 2013 \\
\hline Orthocladius rhyacobius & IDCHI & Orthocladiinae & 1 & & Moller Pillot 2013 \\
\hline Parakiefferiella bathophila & IDCHI & Orthocladiinae & & 1 & Moller Pillot 2013 \\
\hline Parametriocnemus stylatus & IDCHI & Orthocladiinae & 1 & & Moller Pillot 2013 \\
\hline Pseudorthocladius curtistylus & IDCHI & Orthocladiinae & & 1 & Moller Pillot 2013 \\
\hline Rheocricotopus chalybeatus & IDCHI & Orthocladiinae & 1 & & Moller Pillot 2013 \\
\hline Rheocricotopus effusus & IDCHI & Orthocladiinae & 1 & & Moller Pillot 2013 \\
\hline Rheocricotopus fuscipes & IDCHI & Orthocladiinae & 2 & & Moller Pillot 2013 \\
\hline Thienemannia & IDCHI & Orthocladiinae & 1 & & Moller Pillot 2013 \\
\hline Thienemanniella clavicornis agg. & IDCHI & Orthocladiinae & 1 & & Moller Pillot 2013 \\
\hline Thienemanniella majuscula & IDCHI & Orthocladiinae & 1 & 1 & Moller Pillot 2013 \\
\hline Tvetenia calvescens agg. & IDCHI & Orthocladiinae & 1 & & Moller Pillot 2013 \\
\hline Tvetenia discoloripes agg. & IDCHI & Orthocladiinae & 2 & 2 & Moller Pillot 2013 \\
\hline Odontomesa fulva & IDCHI & Prodiamesinae & 2 & & Moller Pillot 2013 \\
\hline Prodiamesa olivacea & IDCHI & Prodiamesinae & 2 & & Moller Pillot 2013 \\
\hline Prodiamesa rufovittata & IDCHI & Prodiamesinae & 1 & & Moller Pillot 2013 \\
\hline Apsectrotanypus trifascipennis & IDCHI & Tanypodinae & & 3 & Vallenduuk \& Moller Pillot 2007 \\
\hline Clinotanypus nervosus & IDCHI & Tanypodinae & 3 & 3 & Vallenduuk \& Moller Pillot 2007 \\
\hline Conchapelopia & IDCHI & Tanypodinae & 2 & 2 & Vallenduuk \& Moller Pillot 2007 \\
\hline Macropelopia adaucta & IDCHI & Tanypodinae & 2 & 2 & Vallenduuk \& Moller Pillot 2007 \\
\hline Macropelopia notata & IDCHI & Tanypodinae & 1 & & Vallenduuk \& Moller Pillot 2007 \\
\hline Paramerina cingulata & IDCHI & Tanypodinae & & 2 & Vallenduuk \& Moller Pillot 2007 \\
\hline Psectrotanypus varius & IDCHI & Tanypodinae & 3 & 3 & Vallenduuk \& Moller Pillot 2007 \\
\hline Trissopelopia longimanus & IDCHI & Tanypodinae & 1 & 1 & Vallenduuk \& Moller Pillot 2007 \\
\hline Micropsectra apposita & IDCHI & Tanytarsini & 2 & & Klink \& Moller Pillot 1996 \\
\hline Micropsectra junci & IDCHI & Tanytarsini & 1 & & Klink \& Moller Pillot 1996 \\
\hline
\end{tabular}




\begin{tabular}{|c|c|c|c|c|c|}
\hline Taxonnaam TWN & $\begin{array}{l}\text { Hoofdgroep } \\
\text { TWN }\end{array}$ & Familie & $\begin{array}{l}\text { R4 } \\
\text { herzien }\end{array}$ & $\begin{array}{l}\text { R4 } \\
\text { origineel }\end{array}$ & Referentie(s) \\
\hline Micropsectra notescens & IDCHI & Tanytarsini & 2 & 2 & Klink \& Moller Pillot 1996 \\
\hline Micropsectra pallidula & IDCHI & Tanytarsini & 1 & 1 & $x$ \\
\hline Micropsectra recurvata & IDCHI & Tanytarsini & 2 & 2 & Klink \& Moller Pillot 1996 \\
\hline Rheotanytarsus & IDCHI & Tanytarsini & 2 & & Klink \& Moller Pillot 1996 \\
\hline Stempellinella brevis & IDCHI & Tanytarsini & 1 & & Ekrem 2007 \\
\hline Stempellinella edwardsi & IDCHI & Tanytarsini & 1 & 1 & Ekrem 2007 \\
\hline Tanytarsus eminulus & IDCHI & Tanytarsini & 1 & & Cuppen et al. 2015 \\
\hline Tanytarsus heusdensis & IDCHI & Tanytarsini & 2 & & Cuppen et al. 2015 \\
\hline Tanytarsus lactescens & IDCHI & Tanytarsini & 1 & & Cuppen et al. 2015 \\
\hline Tanytarsus palettaris & IDCHI & Tanytarsini & 1 & & Cuppen et al. 2015 \\
\hline Atrichops crassipes & IDREM & Athericidae & 1 & & $x$ \\
\hline Chaoborus crystallinus & IDREM & Chaoboridae & 3 & 3 & $x$ \\
\hline Chaoborus flavicans & IDREM & Chaoboridae & 3 & & $x$ \\
\hline Culicidae & IDREM & Culicidae & 3 & 3 & $\mathrm{x}$ \\
\hline Dixa & IDREM & Dixidae & 1 & & Disney 1999 \\
\hline Clinocera & IDREM & Empididae & 1 & & Faasch 2015 \\
\hline Limoniidae & IDREM & Limoniidae & 2 & & $x$ \\
\hline Limnophora & IDREM & Muscidae & 1 & 1 & Merritt \& Wotton 1988 \\
\hline Dicranota & IDREM & Pediciidae & 2 & & $x$ \\
\hline Pedicia & IDREM & Pediciidae & 1 & & $x$ \\
\hline Pedicia rivosa & IDREM & Pediciidae & & 1 & $\mathrm{x}$ \\
\hline Pneumia nubila & IDREM & Psychodidae & & 1 & $\mathrm{x}$ \\
\hline Chrysops caecutiens & IDREM & Tabanidae & 1 & & Zeegers \& van haaren 2000 \\
\hline Simulium angustipes & IDSIM & Simuliidae & 1 & 1 & Lock \& van Maanen 2014 \\
\hline Simulium aureum & IDSIM & Simuliidae & 1 & 1 & Lock \& van Maanen 2014 \\
\hline Simulium costatum & IDSIM & Simuliidae & 1 & 1 & Lock \& van Maanen 2014 \\
\hline Simulium cryophilum & IDSIM & Simuliidae & 1 & 1 & Lock \& van Maanen 2014 \\
\hline Simulium erythrocephalum & IDSIM & Simuliidae & 2 & 1 & Lock \& van Maanen 2014 \\
\hline
\end{tabular}




\begin{tabular}{|c|c|c|c|c|c|}
\hline Taxonnaam TWN & $\begin{array}{l}\text { Hoofdgroep } \\
\text { TWN }\end{array}$ & Familie & $\begin{array}{l}\text { R4 } \\
\text { herzien }\end{array}$ & $\begin{array}{l}\mathrm{R4} \\
\text { origineel }\end{array}$ & Referentie(s) \\
\hline Simulium latipes & IDSIM & Simuliidae & & 1 & Lock \& van Maanen 2014 \\
\hline Simulium Iundstromi & IDSIM & Simuliidae & 1 & & Lock \& van Maanen 2014 \\
\hline Simulium morsitans & IDSIM & Simuliidae & 1 & 1 & Lock \& van Maanen 2014 \\
\hline Simulium noelleri & IDSIM & Simuliidae & 2 & & Lock \& van Maanen 2014 \\
\hline Simulium ornatum & IDSIM & Simuliidae & & 2 & $x$ \\
\hline Simulium ornatum gr. & IDSIM & Simuliidae & 2 & & Lock \& van Maanen 2014 \\
\hline Simulium trifasciatum & IDSIM & Simuliidae & & 2 & Lock \& van Maanen 2014 \\
\hline Simulium vernum & IDSIM & Simuliidae & 1 & 1 & Lock \& van Maanen 2014 \\
\hline Pomatinus substriatus & INCOL & Dryopidae & 1 & & med. Barend van Maanen \\
\hline Agabus biguttatus & INCOL & Dytiscidae & & 1 & Drost et al. 1992 \\
\hline Agabus didymus & INCOL & Dytiscidae & 1 & & Drost et al. 1992 \\
\hline Agabus guttatus & INCOL & Dytiscidae & 1 & 1 & Drost et al. 1992 \\
\hline Agabus paludosus & INCOL & Dytiscidae & 1 & & Drost et al. 1992 \\
\hline Agabus striolatus & INCOL & Dytiscidae & & 1 & Drost et al. 1992 \\
\hline Deronectes latus & INCOL & Dytiscidae & 1 & 1 & Drost et al. 1992 \\
\hline Dytiscus semisulcatus & INCOL & Dytiscidae & 1 & & Drost et al. 1992 \\
\hline Hydroporus discretus & INCOL & Dytiscidae & 1 & 1 & Drost et al. 1992 \\
\hline Hydroporus longulus & INCOL & Dytiscidae & 1 & 1 & Drost et al. 1992 \\
\hline Hydroporus memnonius & INCOL & Dytiscidae & 1 & & Drost et al. 1992 \\
\hline Hydroporus nigrita & INCOL & Dytiscidae & 1 & 1 & Drost et al. 1992 \\
\hline Ilybius chalconatus & INCOL & Dytiscidae & 1 & & Drost et al. 1992 \\
\hline Nebrioporus elegans & INCOL & Dytiscidae & 1 & & Drost et al. 1992 \\
\hline Oreodytes sanmarkii & INCOL & Dytiscidae & 1 & 1 & Drost et al. 1992 \\
\hline Platambus maculatus & INCOL & Dytiscidae & 1 & & Drost et al. 1992 \\
\hline Scarodytes halensis & INCOL & Dytiscidae & 1 & 1 & med. Barend van Maanen \\
\hline Stictotarsus duodecimpustulatus & INCOL & Dytiscidae & 1 & & Drost et al. 1992 \\
\hline Elmis aenea & INCOL & Elmidae & 1 & 1 & Drost et al. 1992 \\
\hline Elmis maugetii & INCOL & Elmidae & 1 & & Drost et al. 1992 \\
\hline
\end{tabular}




\begin{tabular}{|c|c|c|c|c|c|}
\hline Taxonnaam TWN & $\begin{array}{l}\text { Hoofdgroep } \\
\text { TWN }\end{array}$ & Familie & $\begin{array}{l}\text { R4 } \\
\text { herzien }\end{array}$ & $\begin{array}{l}\mathrm{R4} \\
\text { origineel }\end{array}$ & Referentie(s) \\
\hline Esolus angustatus & INCOL & Elmidae & & 1 & Drost et al. 1992 \\
\hline Esolus pygmaeus & INCOL & Elmidae & & 1 & Drost et al. 1992 \\
\hline Limnius volckmari & INCOL & Elmidae & 1 & 1 & Drost et al. 1992 \\
\hline Oulimnius tuberculatus & INCOL & Elmidae & 1 & & Drost et al. 1992 \\
\hline Riolus cupreus & INCOL & Elmidae & & 1 & Drost et al. 1992 \\
\hline Riolus subviolaceus & INCOL & Elmidae & & 1 & Drost et al. 1992 \\
\hline Gyrinus substriatus & INCOL & Gyrinidae & & 1 & Drost et al. 1992 \\
\hline Orectochilus villosus & INCOL & Gyrinidae & 1 & 1 & Drost et al. 1992 \\
\hline Brychius elevatus & INCOL & Haliplidae & 1 & 1 & Drost et al. 1992 \\
\hline Haliplus laminatus & INCOL & Haliplidae & 1 & & Drost et al. 1992 \\
\hline Haliplus sibiricus & INCOL & Haliplidae & 1 & & Drost et al. 1992 \\
\hline Helophorus arvernicus & INCOL & Helophoridae & 1 & 1 & Drost et al. 1992 \\
\hline Hydraena assimilis & INCOL & Hydraenidae & 1 & & Drost et al. 1992 \\
\hline Hydraena excisa & INCOL & Hydraenidae & 1 & 1 & Drost et al. 1992 \\
\hline Hydraena melas & INCOL & Hydraenidae & 1 & & Drost et al. 1992 \\
\hline Hydraena pulchella & INCOL & Hydraenidae & 1 & 1 & Drost et al. 1992 \\
\hline Hydraena riparia & INCOL & Hydraenidae & 1 & 1 & Drost et al. 1992 \\
\hline Limnebius truncatellus & INCOL & Hydraenidae & 1 & 1 & Drost et al. 1992 \\
\hline Ochthebius bicolon & INCOL & Hydraenidae & 1 & & Drost et al. 1992 \\
\hline Hydrochus angustatus & INCOL & Hydrochidae & 1 & 1 & Drost et al. 1992 \\
\hline Chaetarthria similis & INCOL & Hydrophilidae & 1 & & med. Barend van Maanen \\
\hline Laccobius atratus & INCOL & Hydrophilidae & 1 & 1 & Drost et al. 1992 \\
\hline Laccobius obscuratus & INCOL & Hydrophilidae & & 1 & Drost et al. 1992 \\
\hline Laccobius sinuatus & INCOL & Hydrophilidae & 1 & 1 & Drost et al. 1992 \\
\hline Laccobius striatulus & INCOL & Hydrophilidae & 1 & 1 & Drost et al. 1992 \\
\hline Elodes & INCOL & Scirtidae & 2 & & Drost 2008 \\
\hline Elodes minuta & INCOL & Scirtidae & & 2 & $x$ \\
\hline Metreletus balcanicus & INEPH & Ameletidae & 1 & & Drukker in prep. \\
\hline
\end{tabular}




\begin{tabular}{|c|c|c|c|c|c|}
\hline Taxonnaam TWN & $\begin{array}{l}\text { Hoofdgroep } \\
\text { TWN }\end{array}$ & Familie & $\begin{array}{l}\text { R4 } \\
\text { herzien }\end{array}$ & $\begin{array}{l}\mathbf{R 4} \\
\text { origineel }\end{array}$ & Referentie(s) \\
\hline Baetis fuscatus & INEPH & Baetidae & 1 & & Drukker in prep. \\
\hline Baetis niger & INEPH & Baetidae & 1 & 1 & Drukker in prep. \\
\hline Baetis rhodani & INEPH & Baetidae & 1 & & Drukker in prep. \\
\hline Baetis vernus & INEPH & Baetidae & 2 & 2 & Drukker in prep. \\
\hline Centroptilum luteolum & INEPH & Baetidae & 1 & & Drukker in prep. \\
\hline Cloeon dipterum & INEPH & Baetidae & 3 & 3 & Drukker in prep. \\
\hline Cloeon simile & INEPH & Baetidae & & 3 & Drukker in prep. \\
\hline Procloeon bifidum & INEPH & Baetidae & 1 & 1 & Drukker in prep. \\
\hline Brachycercus harrisella & INEPH & Caenidae & 1 & & Drukker in prep. \\
\hline Caenis horaria & INEPH & Caenidae & & 3 & Drukker in prep. \\
\hline Caenis pseudorivulorum & INEPH & Caenidae & 1 & & Drukker in prep. \\
\hline Serratella ignita & INEPH & Ephemerellidae & 1 & 1 & Drukker in prep. \\
\hline Ephemera danica & INEPH & Ephemeridae & 1 & 1 & Drukker in prep. \\
\hline Heptagenia flava & INEPH & Heptageniidae & 1 & & Drukker in prep. \\
\hline Rhithrogena semicolorata & INEPH & Heptageniidae & & 1 & Drukker in prep. \\
\hline Habrophlebia fusca & INEPH & Leptophlebiidae & 1 & & Drukker in prep. \\
\hline Leptophlebia marginata & INEPH & Leptophlebiidae & 1 & 1 & Drukker in prep. \\
\hline Paraleptophlebia submarginata & INEPH & Leptophlebiidae & 1 & & Drukker in prep. \\
\hline Siphlonurus aestivalis & INEPH & Siphlonuridae & 1 & 1 & Drukker in prep. \\
\hline Siphlonurus armatus & INEPH & Siphlonuridae & 1 & 1 & Drukker in prep. \\
\hline Micronecta poweri & INHET & Corixidae & 1 & 1 & Aukema et al. 2002; Tempelman \& van Haaren 2009 \\
\hline Sigara hellensii & INHET & Corixidae & 1 & 1 & Aukema et al. 2002; Tempelman \& van Haaren 2009 \\
\hline Aquarius najas & INHET & Gerridae & 1 & 1 & Aukema et al. 2002; Tempelman \& van Haaren 2009 \\
\hline Gerris gibbifer & INHET & Gerridae & & 1 & Aukema et al. 2002; Tempelman \& van Haaren 2009 \\
\hline Notonecta maculata & INHET & Notonectidae & 1 & & Aukema et al. 2002; Tempelman \& van Haaren 2009 \\
\hline Velia caprai & INHET & Veliidae & 1 & 1 & Aukema et al. 2002; Tempelman \& van Haaren 2009 \\
\hline Velia saulii & INHET & Veliidae & 1 & 1 & Aukema et al. 2002; Tempelman \& van Haaren 2009 \\
\hline Aeshna juncea & INODO & Aeshnidae & & 1 & www.libellennet.nl \\
\hline
\end{tabular}




\begin{tabular}{|c|c|c|c|c|c|}
\hline Taxonnaam TWN & $\begin{array}{l}\text { Hoofdgroep } \\
\text { TWN }\end{array}$ & Familie & $\begin{array}{l}\text { R4 } \\
\text { herzien }\end{array}$ & $\begin{array}{l}\mathrm{R4} \\
\text { origineel }\end{array}$ & Referentie(s) \\
\hline Calopteryx splendens & INODO & Calopterygidae & 2 & & www.libellennet.nl \\
\hline Calopteryx virgo & INODO & Calopterygidae & 1 & 1 & www.libellennet.nl \\
\hline Ceriagrion tenellum & INODO & Coenagrionidae & & 1 & www.libellennet.nl \\
\hline Coenagrion mercuriale & INODO & Coenagrionidae & 1 & & www.libellennet.nl \\
\hline Cordulegaster boltonii & INODO & Cordulegastridae & 1 & 1 & www.libellennet.nl \\
\hline Orthetrum coerulescens & INODO & Libellulidae & 1 & & www.libellennet.nl \\
\hline Sympetrum pedemontanum & INODO & Libellulidae & 1 & & www.libellennet.nl \\
\hline Platycnemis pennipes & INODO & Platycnemididae & 1 & & www.libellennet.nl \\
\hline Leuctra fusca & INREM & Leuctridae & 1 & 1 & Koese 2008 \\
\hline Leuctra nigra & INREM & Leuctridae & 1 & 1 & Koese 2008 \\
\hline Amphinemura standfussi & INREM & Nemouridae & 1 & 1 & Koese 2008 \\
\hline Amphinemura sulcicollis & INREM & Nemouridae & & 1 & Koese 2008 \\
\hline Nemoura avicularis & INREM & Nemouridae & 1 & 1 & Koese 2008 \\
\hline Nemoura cinerea & INREM & Nemouridae & 2 & & Koese 2008 \\
\hline Nemoura dubitans & INREM & Nemouridae & 1 & 1 & Koese 2008 \\
\hline Nemoura marginata & INREM & Nemouridae & 1 & 1 & Koese 2008 \\
\hline Nemurella pictetii & INREM & Nemouridae & 1 & 1 & Koese 2008 \\
\hline Isoperla grammatica & INREM & Perlodidae & 1 & 1 & Koese 2008 \\
\hline Sisyra & INREM & Neuroptera & & 1 & Elliott 2009 \\
\hline Osmylus fulvicephalus & INREM & Osmylidae & 1 & 1 & Elliott 2009 \\
\hline Sialis fuliginosa & INREM & Sialidae & 1 & 1 & Elliott 2009 \\
\hline Sialis Iutaria & INREM & Sialidae & & 3 & Elliott 2009 \\
\hline Apatania fimbriata & INTRI & Apataniidae & 1 & 1 & Higler 2008 \\
\hline Beraea maurus & INTRI & Beraeidae & 1 & 1 & Higler 2008 \\
\hline Beraea pullata & INTRI & Beraeidae & 1 & & Higler 2008 \\
\hline Beraeodes minutus & INTRI & Beraeidae & 1 & 1 & Higler 2008 \\
\hline Agapetus fuscipes & INTRI & Glossosomatidae & 1 & 1 & Higler 2008 \\
\hline Goera pilosa & INTRI & Goeridae & 1 & 1 & Higler 2008 \\
\hline
\end{tabular}




\begin{tabular}{|c|c|c|c|c|c|}
\hline Taxonnaam TWN & $\begin{array}{l}\text { Hoofdgroep } \\
\text { TWN }\end{array}$ & Familie & $\begin{array}{l}\text { R4 } \\
\text { herzien }\end{array}$ & $\begin{array}{l}\text { R4 } \\
\text { origineel }\end{array}$ & Referentie(s) \\
\hline Lithax obscurus & INTRI & Goeridae & 1 & & Higler 2008 \\
\hline Silo nigricornis & INTRI & Goeridae & 1 & 1 & Higler 2008 \\
\hline Silo pallipes & INTRI & Goeridae & 1 & & Higler 2008 \\
\hline Hydropsyche angustipennis & INTRI & Hydropsychidae & 1 & 2 & Higler 2008 \\
\hline Hydropsyche fulvipes & INTRI & Hydropsychidae & & 1 & Higler 2008 \\
\hline Hydropsyche instabilis & INTRI & Hydropsychidae & 1 & & Higler 2008 \\
\hline Hydropsyche pellucidula & INTRI & Hydropsychidae & 1 & 1 & Higler 2008 \\
\hline Hydropsyche saxonica & INTRI & Hydropsychidae & 1 & 1 & Higler 2008 \\
\hline Hydropsyche siltalai & INTRI & Hydropsychidae & 1 & & Higler 2008 \\
\hline Hydroptila & INTRI & Hydroptilidae & 1 & & Higler 2008 \\
\hline Ithytrichia lamellaris & INTRI & Hydroptilidae & 1 & 1 & Higler 2008 \\
\hline Ptilocolepus granulatus & INTRI & Hydroptilidae & 1 & 1 & Higler 2008 \\
\hline Crunoecia irrorata & INTRI & Lepidostomatidae & 1 & 1 & Higler 2008 \\
\hline Lasiocephala basalis & INTRI & Lepidostomatidae & 1 & 1 & Higler 2008 \\
\hline Adicella reducta & INTRI & Leptoceridae & 1 & 1 & Higler 2008 \\
\hline Athripsodes cinereus & INTRI & Leptoceridae & 2 & & Higler 2008 \\
\hline Anabolia nervosa & INTRI & Limnephilidae & 2 & 1 & Higler 2008 \\
\hline Annitella obscurata & INTRI & Limnephilidae & 1 & & Higler 2008 \\
\hline Chaetopteryx villosa & INTRI & Limnephilidae & 1 & & Higler 2008 \\
\hline Drusus annulatus & INTRI & Limnephilidae & & 1 & Higler 2008 \\
\hline Enoicyla pusilla & INTRI & Limnephilidae & 1 & & Higler 2008 \\
\hline Glyphotaelius pellucidus & INTRI & Limnephilidae & 1 & & Higler 2008 \\
\hline Grammotaulius submaculatus & INTRI & Limnephilidae & & 1 & Higler 2008 \\
\hline Halesus digitatus & INTRI & Limnephilidae & & 1 & Higler 2008 \\
\hline Halesus & INTRI & Limnephilidae & 1 & & Higler 2008 \\
\hline Hydatophylax infumatus & INTRI & Limnephilidae & 1 & & Higler 2008 \\
\hline Ironoquia dubia & INTRI & Limnephilidae & 1 & 1 & Higler 2008 \\
\hline Limnephilus binotatus & INTRI & Limnephilidae & & 1 & Higler 2008 \\
\hline
\end{tabular}




\begin{tabular}{|c|c|c|c|c|c|}
\hline Taxonnaam TWN & $\begin{array}{l}\text { Hoofdgroep } \\
\text { TWN }\end{array}$ & Familie & $\begin{array}{l}\text { R4 } \\
\text { herzien }\end{array}$ & $\begin{array}{l}\text { R4 } \\
\text { origineel }\end{array}$ & Referentie(s) \\
\hline Limnephilus centralis & INTRI & Limnephilidae & 1 & 1 & Higler 2008 \\
\hline Limnephilus elegans & INTRI & Limnephilidae & & 1 & Higler 2008 \\
\hline Limnephilus extricatus & INTRI & Limnephilidae & 1 & 1 & Higler 2008 \\
\hline Limnephilus fuscicornis & INTRI & Limnephilidae & 1 & 1 & Higler 2008 \\
\hline Limnephilus griseus & INTRI & Limnephilidae & & 1 & Higler 2008 \\
\hline Limnephilus lunatus & INTRI & Limnephilidae & 2 & 3 & Higler 2008 \\
\hline Limnephilus stigma & INTRI & Limnephilidae & & 1 & Higler 2008 \\
\hline Limnephilus subcentralis & INTRI & Limnephilidae & & 1 & Higler 2008 \\
\hline Micropterna lateralis & INTRI & Limnephilidae & 1 & 1 & Higler 2008 \\
\hline Micropterna sequax & INTRI & Limnephilidae & 1 & 1 & Higler 2008 \\
\hline Potamophylax cingulatus & INTRI & Limnephilidae & 1 & 1 & Higler 2008 \\
\hline Potamophylax latipennis & INTRI & Limnephilidae & 1 & 1 & Higler 2008 \\
\hline Potamophylax luctuosus & INTRI & Limnephilidae & 1 & 1 & Higler 2008 \\
\hline Potamophylax nigricornis & INTRI & Limnephilidae & 1 & & Higler 2008 \\
\hline Potamophylax rotundipennis & INTRI & Limnephilidae & 1 & & Higler 2008 \\
\hline Rhadicoleptus alpestris & INTRI & Limnephilidae & & 1 & Higler 2008 \\
\hline Stenophylax permistus & INTRI & Limnephilidae & 1 & 1 & Higler 2008 \\
\hline Molanna angustata & INTRI & Molannidae & 1 & & Higler 2008 \\
\hline Molannodes tinctus & INTRI & Molannidae & 1 & & Higler 2008 \\
\hline Wormaldia occipitalis & INTRI & Philopotamidae & 1 & 1 & Higler 2008 \\
\hline Wormaldia subnigra & INTRI & Philopotamidae & 1 & 1 & Higler 2008 \\
\hline Trichostegia minor & INTRI & Phryganeidae & & 1 & Higler 2008 \\
\hline Plectrocnemia conspersa & INTRI & Polycentropodidae & 1 & & Higler 2008 \\
\hline Polycentropus flavomaculatus & INTRI & Polycentropodidae & & 1 & Higler 2008 \\
\hline Polycentropus irroratus & INTRI & Polycentropodidae & 1 & & Higler 2008 \\
\hline Tinodes unicolor & INTRI & Polycentropodidae & 1 & 1 & Higler 2008 \\
\hline Lype & INTRI & Psychomyiidae & 1 & & Higler 2008 \\
\hline Tinodes assimilis & INTRI & Psychomyiidae & 1 & 1 & Higler 2008 \\
\hline
\end{tabular}




\begin{tabular}{|c|c|c|c|c|c|}
\hline Taxonnaam TWN & $\begin{array}{l}\text { Hoofdgroep } \\
\text { TWN }\end{array}$ & Familie & $\begin{array}{l}\text { R4 } \\
\text { herzien }\end{array}$ & $\begin{array}{l}\mathbf{R 4} \\
\text { origineel }\end{array}$ & Referentie(s) \\
\hline Tinodes pallidulus & INTRI & Psychomyiidae & 1 & 1 & Higler 2008 \\
\hline Rhyacophila dorsalis & INTRI & Rhyacophilidae & 1 & & Higler 2008 \\
\hline Rhyacophila fasciata & INTRI & Rhyacophilidae & 1 & 1 & Higler 2008 \\
\hline Notidobia ciliaris & INTRI & Sericostomatidae & 1 & 1 & Higler 2008 \\
\hline Sericostoma personatum & INTRI & Sericostomatidae & 1 & & Higler 2008 \\
\hline Oxyethira & INTRI & Trichoptera & & 1 & Higler 2008 \\
\hline Pisidium personatum & MOBIV & Sphaeriidae & 1 & & Gittenberger \& Janssen 1998; Killeen et al. 2004 \\
\hline Pisidium pulchellum & MOBIV & Sphaeriidae & 2 & & Gittenberger \& Janssen 1998; Killeen et al. 2004 \\
\hline Sphaerium corneum & MOBIV & Sphaeriidae & 3 & 3 & Gittenberger \& Janssen 1998; Killeen et al. 2004 \\
\hline Ancylus fluviatilis & MOGAS & Ancylidae & 1 & & Gittenberger \& Janssen (1998) \\
\hline Bithynia leachi & MOGAS & Bithyniidae & 3 & 3 & Gittenberger \& Janssen (1998) \\
\hline Bithynia tentaculata & MOGAS & Bithyniidae & 3 & 3 & Gittenberger \& Janssen (1998) \\
\hline Radix balthica gr. & MOGAS & Lymnaeidae & 3 & 3 & Gittenberger \& Janssen (1998) \\
\hline Stagnicola palustris & MOGAS & Lymnaeidae & & 3 & Gittenberger \& Janssen (1998) \\
\hline Physella acuta & MOGAS & Physidae & 3 & 3 & Gittenberger \& Janssen (1998) \\
\hline Anisus leucostoma/spirorbis & MOGAS & Planorbidae & & 3 & Gittenberger \& Janssen (1998) \\
\hline Anisus vortex & MOGAS & Planorbidae & 3 & 3 & Gittenberger \& Janssen (1998) \\
\hline Bathyomphalus contortus & MOGAS & Planorbidae & 3 & 3 & Gittenberger \& Janssen (1998) \\
\hline Gyraulus albus & MOGAS & Planorbidae & 3 & 3 & Gittenberger \& Janssen (1998) \\
\hline Planorbarius corneus & MOGAS & Planorbidae & 3 & 3 & Gittenberger \& Janssen (1998) \\
\hline Planorbis planorbis & MOGAS & Planorbidae & 3 & 3 & Gittenberger \& Janssen (1998) \\
\hline Valvata cristata & MOGAS & Valvatidae & & 3 & Gittenberger \& Janssen (1998) \\
\hline Valvata macrostoma & MOGAS & Valvatidae & & 3 & Gittenberger \& Janssen (1998) \\
\hline Valvata piscinalis & MOGAS & Valvatidae & 3 & 3 & Gittenberger \& Janssen (1998) \\
\hline
\end{tabular}

Bijbehorende referenties indicatiewaarden:

Aukema, B., Cuppen, J.G.M., Nieser, N. \& D. Tempelman (2002) Verspreidingsatlas Nederlandse wantsen (Hemiptera: Heteroptera) Deel I: Dipsocoromorpha, Nepomorpha, Gerromorpha \& Leptopodomorpha. European Invertebrate Survey - Nederland, Leiden. 
Bartsch, I., Deichsel, R. (2007) Chelicerata: Araneae/Acari. Spektrum Akademischer verlag, Heidelberg.

Cuppen, H., Tempelman, D,, van Haaren T. (2015) Key for identification of 4th instar larvae of Tanytarsus Van der Wulp, 1874 of north-western

Europe (Diptera: Chironomidae: Tanytarsini) Lauterbornia 79: 1-21.

Den Hartog C (1962) De Nederlandse platwormen -Tricladida. Wetenschappelijke mededelingen KNNV 42. KNNV Hoogwoud

Di Sabatino, A., Gerecke, R., Geldhill, T., Smit, H. (2010) Acari: Hydrachnidia II. Spektrum Akademischer verlag, Heidelberg

Disney, R.H.L. (1999) British Dixidae (Meniscus midges) and Thaumaleidae (trickle midges): keys with ecological notes. Freshwater Biological

Association, Ambleside.

Drost, B. (2008) De genera Odeles en Elodes (Coleoptera: Scirtidae) in Nederland. Entomologische Berichten 68 (1): $1212-16$.

Drost, M.B.P., Cuppen, H.P.J.J., van Nieukerken, E.J. \& M. Schreijer (1992). De waterkevers van Nederland. KNNV Uitgeverij, Utrecht.

Drukker, D. De haften van Nederland en België, in prep.

Ekrem T. (2007) A taxonomic revision of the genus Stempellinella (Diptera: Chironomidae), Journal of Natural History 41:21-24.

Elliot, J.M. (2009) Freshwater megaloptera and neuroptera of Brittain en Ireland: keys to aduls and larvae, and a review of their ecology.

Freshwater Biological Association Scientific publication no. 65. Ambleside.

Faasch, H. (2015). Identification guide to aquatic and semi-aquatic Diptera larvae. DGL-Arbeitshilfe 1-2015

Gerecke, R., Geldhill, T., Pesic, V., Smit, H. (2016) Chelicerata: Acari III. Springer-Verlag, Berlin Heidelberg.

Gittenberger, E. \& A.W. Janssen (1998) De Nederlandse zoetwatermollusken. Recente en fossiele weekdieren uit zoet en brak water.

Nederlandse fauna 2. Nationaal Natuurhistorisch museum Leiden, KNNV uitgeverij, EIS-Nederland.

Higler L.W.G. (2008) Verspreidingsatlas Nederlandse kokerjuffers (Trichoptera) EIS-Nederland, Leiden.

Killeen, I., Aldridge, D.C., Oliver, G. (2004) Freshwater Bivalves of Britain and Ireland. FSC Publications, Shrewsbury.

Klink, A., Moller Pilot, H. (1996) Lijst van de Nederlandse Chironomidae bijgewerkt tot I januari 1996. Themanummer WEW-08. Werkgroep

Ecologisch Waterbeheer.

Koese, B. (2008) De Nederlandse steenvliegen (Plecoptera) Entomologische tabellen I, EIS-Nederland

Lock, K. van Maanen, b. (2014) De kriebelmuggen van nederland en vlaanderen (Diptera: Simuliidae) Nederlandse Faunistische Mededelingen 43.

Merritt, R.W., Wotton R.S. (1988) The Life History and Behavior of Limnophora riparia (Diptera:Muscidae), a Predator of Larval Black Flies.

Journal of the North American Benthological Society 7: 1-12

Moller-Pilot, H.K.M. (2009) Chironomidae larvae. Biology and ecology of the Chironomini. KNNV Publishing, Zeist.

Moller-Pilot, H.K.M. (2013) Chironomidae larvae. Biology and ecology of the aquatic Orthocladiinae. KNNV Publishing, Zeist.

Reynoldson T.B., Young J.O. (2000) A Key to the Freshwater Triclads of Brtain and Ireland, With Notes on Their Ecology. Freshwater biological association scientific publication no. 58. Freshwater Biological Association, Ambleside.

Scheers, K., Mertens, J., Thys, N. (2014) On the occurrence of Suphrodytes dorsalis (Fabricius, 1787) and Suphrodytes figuratus (Gyllenhal, 1826) (Coleoptera: Dytiscidae) in Belgium with notes on the habitat. Bulletin van de Koninklijke Belgische Vereniging voor Entomologie 150: 187-189

Smit, H \& H. van der Hammen (2000) Atlas van de Nederlandse watermijten (Acari: Hydracarina). Nederlandse faunistische mededelingen 13: $1-273$. 
Soes, D.M., 2009. De ovale hoornschaal (Sphaerium ovale) ook in Nederland. Spirula 366: 9-10.

Tempelman, D \& T. van Haaren (2009) Water- en oppervlaktewantsen van Nederland. Jeugdbondsuitgeverij, Utrecht.

Vallenduuk, H. Moller-Pilot, H.K.M. (2007) Chironomidae larvae. General ecology and Tanypodinae. KNNV Publishing, Zeist.

van Haaren, T., Hop, H., Soes, M., Tempelman, D. (2004) The freshwater leeches (Hirudinea) of the Netherlands. Lauterbornia 52: 113-131. van Haaren, T., Soors J. (2013) Aquatic Oligochaetes of the Netherlands and Belgium. KNNV Publishing, Zeist.

Zeegers, T., van Haaren, T. (2000) Dazen en dazenlarven : inleiding tot en tabellen voor de Tabanidae (Diptera) van Nederland en België. KNNV Uitgeverij Utrecht. 